\title{
Chaotic advection in a steady, three-dimensional, Ekman-driven eddy
}

\author{
L. J. Pratt ${ }^{1, \dagger}$, I. I. Rypina ${ }^{1}$, T. M. Özgökmen ${ }^{2}$, P. Wang ${ }^{2}$, H. Childs ${ }^{3}$ \\ and Y. Bebieva ${ }^{4}$ \\ ${ }^{1}$ Woods Hole Oceanographic Institution, Woods Hole, MA 02543, USA \\ ${ }^{2}$ Rosenstiel School of Marine and Atmospheric Science, University of Miami, Miami, FL, 33149-1098, \\ USA \\ ${ }^{3}$ Lawrence Berkeley National Laboratory, CA 94720, USA \\ ${ }^{4}$ Graduate School of Arts and Science, Yale University, New Haven, CT, 06520, USA
}

(Received 17 September 2012; revised 16 October 2013; accepted 30 October 2013; first published online 5 December 2013)

We investigate and quantify stirring due to chaotic advection within a steady, three-dimensional, Ekman-driven, rotating cylinder flow. The flow field has vertical overturning and horizontal swirling motion, and is an idealization of motion observed in some ocean eddies. The flow is characterized by strong background rotation, and we explore variations in Ekman and Rossby numbers, $E$ and $R_{o}$, over ranges appropriate for the ocean mesoscale and submesoscale. A high-resolution spectral element model is used in conjunction with linear analytical theory, weakly nonlinear resonance analysis and a kinematic model in order to map out the barriers, manifolds, resonance layers and other objects that provide a template for chaotic stirring. As expected, chaos arises when a radially symmetric background state is perturbed by a symmetrybreaking disturbance. In the background state, each trajectory lives on a torus and some of the latter survive the perturbation and act as barriers to chaotic transport, a result consistent with an extension of the KAM theorem for three-dimensional, volume-preserving flow. For shallow eddies, where $E$ is $O(1)$, the flow is dominated by thin resonant layers sandwiched between KAM-type barriers, and the stirring rate is weak. On the other hand, eddies with moderately small $E$ experience thicker resonant layers, wider-spread chaos and much more rapid stirring. This trend reverses for sufficiently small $E$, corresponding to deep eddies, where the vertical rigidity imposed by strong rotation limits the stirring. The bulk stirring rate, estimated from a passive tracer release, confirms the non-monotonic variation in stirring rate with $E$. This result is shown to be consistent with linear Ekman layer theory in conjunction with a resonant width calculation and the Taylor-Proudman theorem. The theory is able to roughly predict the value of $E$ at which stirring is maximum. For large disturbances, the stirring rate becomes monotonic over the range of Ekman numbers explored. We also explore variation in the eddy aspect ratio.

Key words: chaotic advection, geophysical and geological flows, ocean processes 


\section{Introduction}

Chaotic advection (Aref 1984) is a process in which fluid stirring is controlled by long-lived, coherent structures. Stirring in such flows is of particular interest as it leads to exponentially rapid stretching and folding of fluid elements, amplification of property gradients and eventually irreversible mixing. However, if chaotic regions exist, it does not mean the entire flow field must be chaotic. Non-chaotic regions, where the stirring is relatively weak, can exist in other portions of the flow field, separated by material barriers.

This picture is very relevant in the stratospheric polar vortex (e.g. Ngan \& Shephard 1999; Joseph \& Legras 2002; Rypina et al. 2007b). In the ocean, the dynamical systems approaches often used to analyse chaotic transport phenomena have been applied to certain features or scales, or to particular geographical areas. Early investigations of idealized jets, waves and gyres (e.g. Sommeria, Meyers \& Swinney 1989; Behringer, Meyers \& Swinney 1991; Samelson 1992; Del-Castillo-Negrete \& Morrison 1993; Pratt, Lozier \& Beliakova 1995; Duan \& Wiggins 1996; Dijkstra \& Katsman 1997; Haller \& Poje 1998) led to applications to the Gulf Stream (Samelson 1992; Lozier et al. 1997; Malhotra \& Wiggins 1998; Rogerson et al. 1999; Poje \& Haller 1999; Deese, Pratt \& Helfrich 2002; Yuan, Pratt \& Jones 2004), flow around islands (Miller et al. 2002), exchange between subtropical and subpolar gyres (Coulliette \& Wiggins 2001), tidal-driven motion in Monterey Bay (Lipphardt et al. 2006; Coulliette et al. 2007; Shadden et al. 2009), mesoscale motion in the Gulf of Mexico (Kruznetsov et al. 2002; Beron-Vera \& Olascoaga 2009; Branicki \& Kirwan 2010), dipoles in the Philippine Archipelago (Rypina et al. 2010) and gyres in the Adriatic Sea (Haza et al. 2008; Rypina, Brown \& Kocak 2009). The methods are also used in the planning of targeted release of drifters (Haza et al. 2007) and in the statistical descriptions of dispersion (Poje et al. 2010). The full extent to which the methods and ideas are relevant is unknown, but the rule-of-thumb applicability criterion is that chaotic advection is relevant when the flow field contains long-lived, coherent structures that control stirring at both large and small scales. As argued by Rypina et al. (2011) this picture does not apply to large basin-scale ocean gyres, motivation for canonical 'double gyre' models, because the influence of mesoscale eddies cannot be ignored.

Most oceanographic applications involve flows that are assumed to be horizontally two-dimensional and non-divergent, either at the upper surface or on surfaces of constant depth or density. Horizontal divergence of the velocity field is ignored or removed. The quasigeostrophic approximation is consistent with this assumption, since the vertical velocity $w$ must be sufficiently weak that its vertical derivative $\partial w / \partial z$ can be neglected to leading order in the continuity equation. For this reason, three-dimensional, quasigeostrophic eddies or jets can be treated by making a twodimensional analysis at each depth or isopycnal surface and piecing the results together (e.g. Branicki \& Kirwan (2010) for stratified Gulf of Mexico eddies). Relatively little attention has been paid to flow fields that are both oceanographically relevant and fully three-dimensional, defined here as meaning that $\partial w / \partial z$ is not negligible at leading order. The number of eigenvalues of the velocity gradient tensor increases from two (both real) to three (one real plus a complex conjugate pair), and the inward or outward spiralling that is prohibited in incompressible, twodimensional applications can arise. In general, the extra degree of freedom would appear to encourage chaotic motion and rapid stirring, and it is natural to ask whether barriers are easily permitted in such flow. The answer is positive, as suggested by special solutions to the Euler equations (e.g. Dombre et al. (1986) for ABC flow) 
or three-dimensional phenomenological models (Solomon \& Mezic 2003; Mullowney, Julian \& Meiss 2005, 2008) and laboratory and numerical experiments (Fountain et al. 2000; Lackey \& Sotiropoulos 2006).

The ocean is filled with eddies of different sizes, many of them long-lived. For coherent eddies that have upwelling and downwelling circulations, the co-existence of strongly and weakly mixed regions, separated by barriers, could have all kinds of implications for the transport, mixing and distribution of physical, chemical and biological quantities and for ecosystems. The vorticity supplied by Earth's rotation is often much larger than that due to the swirling motion in these eddies, and this places the flow fields in a different dynamical regime than previous three-dimensional models of chaotic advection. Although previous work has identified the generic forms, usually tori, that barriers can take, their size, stability and distribution has not been evaluated in an oceanographically relevant parameter space. Nor do we know the extent to which chaos enhances the stirring rates within this space. Some guidance follows from the KAM theorem and the analysis of resonant layers, but these are based only on the underlying geometry of the given incompressible velocity field. The dynamical constraints that forge the velocity field do not inform the theorem and are often ignored in modern analysis of Lagrangian coherent structures.

The present work represents only a first step towards establishment of the relevance of chaotic advection to eddies in an oceanographically relevant parameter range. We analyse a dynamically consistent, three-dimensional flow field that is both simple and canonical but also lies within a parameter range spanning the ocean submesoscale to the mesoscale. The specific flow field has a horizontal swirling circulation plus an overturning cell, all driven by an imposed velocity at the upper surface. The curl of the surface stress leads to a divergence in the surface Ekman layer and induced upwelling and downwelling. This type of circulation has been observed, for example, in mesoscale eddies subject to wind forcing (McGillicuddy et al. 2007; Ledwell, McGillicuddy \& Anderson 2008). Stratification is ignored, but this is reasonable for submesocale feature that reside primarily in the mixed layer, and may be informative for mode water eddies (Ledwell et al. 2008), which tend to be weakly stratified. For a basic state with no azimuthal dependence, it is readily established that all trajectories are confined to tori and no trajectory is chaotic. As demonstrated by Fountain et al. (2000), chaos can be introduced into the system through a steady, symmetry destroying perturbation. We describe the Lagrangian structure of the resulting flow with respect to chaotic and non-chaotic (regular) regions and the boundaries that separate the two. We will map out general trends in the space of the primary governing parameters (the Ekman and Rossby numbers, the aspect ratio and the disturbance amplitude) paying attention to structural characteristics and barrier stability. The amplification of the stirring rates due to the Lagrangian chaos will be quantified through analysis of tracer release experiments. We will show that for a weak or moderate disturbance, the stirring rate is a non-monotonic function of the Ekman number, which agrees with earlier predictions due to Mezic (2001). However, we will also argue that the dynamical explanation differs from what is thought to apply in flows that lack strong background rotation and that the non-monotonic behaviour disappears when the disturbance amplitude grows sufficiently large. We will also explore variations in the Rossby number and aspect ratio of the flow. We will also use various analytical results, including linear Ekman layer solutions, the Taylor-Proudman theorem and a calculation of resonance width, to construct a narrative explaining many, although not all, of the observed transitions and regimes. 
Examples of invariant tori have been visualized using dye in the beautiful laboratory experiments (Fountain et al. 2000) in which a viscous flow in a cylinder is forced at the top by a tilted, rotating impellor. Most of the cases considered lack background rotation. Lackey \& Sotiropoulos (2006) also consider cylinder flow with counterrotating top and bottom, producing a twin overturning circulations. No cases with background rotation are considered. Our study is distinguished from these earlier studies by, among other things, the consideration of oceanographically important frame rotation, which is reflected in the parameter space we explore: Rossby numbers that are low to moderate and Ekman numbers ranging from $O(1)$ to $10^{-4}$.

Our investigation is centred on a high-resolution integration of the Navier-Stokes equations, and this is described in the next section along with the governing parameters and a complementary phenomenological model. Section 3 describes the two mechanisms by which Lagrangian chaos can arise as a result of perturbation of an azimuthally uniform steady state in which each trajectory lives on a torus. Some of this material consists of review (action-angle-angle coordinates, the KAM theorem, stable and unstable manifolds) while other aspects (resonance width calculation) are new. Section 4 describes an exploration of numerical results over variations of Ekman and Rossby numbers and disturbance amplitude. Chaos typically occurs near the rotation axis and outer regions of the cylinder, and in resonant layers, but the geometry and distribution of these objects differs markedly as the Ekman number is varied. The non-monotonic behaviour with $E$ of the vertical velocity, as predicted by linear theory, provides insight into three regimes of distinct geometry and mixing that are identified. In $\S 5$ we present estimates of the bulk stirring rate and use ideas relating to resonance width, the Taylor-Proudman theorem and analytical solutions to construct a narrative and theory that predicts the non-monotonic character of the stirring for weak disturbances. We then describe some significant changes that occur in geometry and mixing rate as the disturbance amplitude is increased. We also comment on some other aspects, including the stability around the central torus, the result of reducing the aspect ratio and the general behaviour of stable and unstable manifolds. Section 6 identifies ocean features and scales for which the stirring mechanisms may be relevant and outlines some observational challenges. Section 7 summarizes the findings and lists some unresolved theoretical issues.

\section{Models for the rotating cylinder flow}

\subsection{Traditional Model with rapid frame rotation}

In a traditional setting (figure 1), the cylinder rotates at angular speed $\Omega$ and is driven by a lid that rotates at speed $\Omega+\delta \Omega$. The linear circulation produced when the Rossby number $R_{o}=|\delta \Omega| / \Omega \ll 1$ is described by Greenspan (1969) and depends on the Ekman number

$$
E=v / \Omega H^{2},
$$

where $v$ is the kinematic viscosity and $H$ is the tank depth. Ekman layers of thickness $(v / \Omega)^{1 / 2}$ exist at the top and bottom of the tank and they are thin compared with $H$ for $E \ll 1$. Relatively thin Ekman layers would be typical for many mesoscale eddies but not necessarily for submesoscale eddies. Between the Ekman layers (and away from the sidewalls) lies an inviscid, geostrophic interior. For ocean applications one should think of $v$ as a turbulent vertical viscosity, producing Ekman layers approximately $20 \mathrm{~m}$ thick. The corresponding value of $E$ would then be $10^{-3}-10^{-4}$ for a deep mesoscale eddy or ring, whose depth might exceed $1000 \mathrm{~m}$, and $O(1)$ for a 


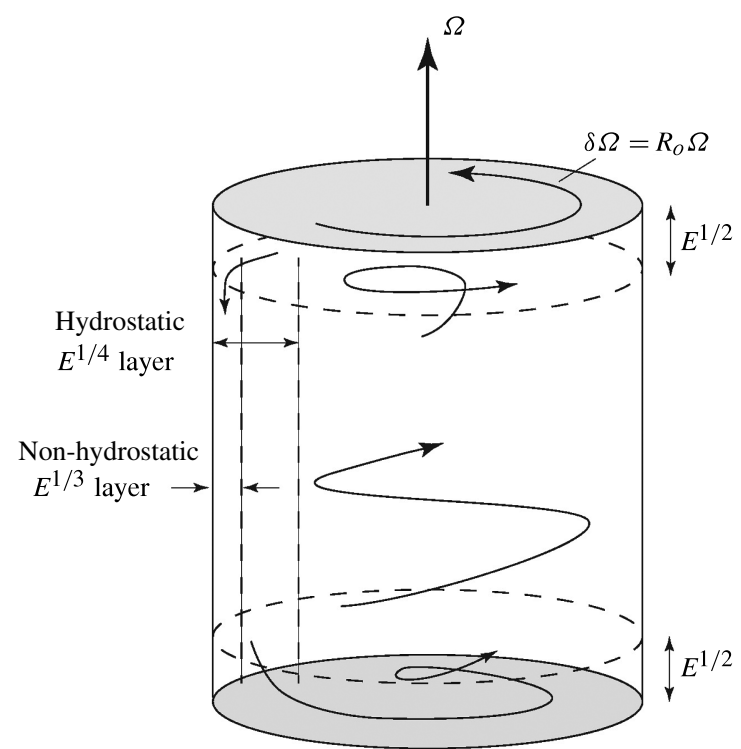

FIGURE 1. (a) The boundary layer structure for the steady, linear flow in a rotating cylinder driven by a differentially rotating lid at low Rossby number. The dimensionless boundary layer thicknesses are given in terms of the Ekman number $E$, which is assumed to be $\ll 1$.

shallow, submesoscale eddy. We will explore this range. Modern models of the upper ocean would use a variable turbulent viscosity or a more sophisticated turbulence closure, but this is beyond the present scope.

The overall picture revealed by the $E \ll 1$ solution is as follows. For $\delta \Omega>0$, the lid produces the equivalent of a cyclonic wind stress at the surface. The top Ekman layer is divergent and sucks up fluid from the geostrophic region below. For small values of the Rossby number

$$
R_{o}=\delta \Omega / \Omega \ll 1
$$

the interior is subject to the Taylor-Proudman theorem, which requires that all three velocity components be independent of $z$. The vertical velocity at the base of the divergent upper Ekman layer must therefore match that at the top of a convergent bottom Ekman layer. It can also be shown that the interior contains a cyclonic, rigidbody circulation, with the azimuthal velocity equal to half the differential lid velocity. The radial velocity in the interior is negligibly small. The volume flux in the top Ekman layer is outward and must be redirected downward when it reaches the outer rim of the can. This takes place largely within a non-hydrostatic, viscous sidewall (Stewardson) layer with dimensionless thickness $E^{1 / 3}$. In addition, there is an $E^{1 / 4}$ layer that participates in the overturning and helps satisfy other boundary conditions. The sidewall layers feed fluid down into the bottom Ekman layer where it is carried radially inward and later up into the geostrophic interior. An asymptotic solution describing the interior and all boundary layers can be written down, but its usefulness for Lagrangian studies is limited by the fact that all trajectories travel through an unresolved $E^{1 / 2} \times E^{1 / 2}$ corner region. (Our literature search has failed to reveal a single source in which the full asymptotic solution is written out, so we have done so at http: //www.whoi.edu/page.do?pid=52816.) These difficulties are relieved if the stress at the 
top surface vanishes at the outer rim. This is achieved in our numerical simulations by replacement of the azimuthal lid velocity $v=\delta \Omega r$ by

$$
v_{\text {lid }}=4 \delta \Omega r(R-r) / R,
$$

where $r$ is the radial coordinate and $\delta \Omega$ now represents the angular speed of the lid at $r=R / 2$. The lid speed vanishes at $r=R$, eliminating the discontinuity and singularity. As shown in the linear solution, written down in appendix $A$, the descending motion now occurs in the broad interior region $2 R / 3<r<R$ rather than in thin sidewall layers.

Variations of the set-up depicted in figure 1 arise in all kinds of industrial and engineering applications. There is a vast literature on the subject, much of it due to Professor J. Lopez and collaborators (see Lopez \& Marques (2010) and references contained therein). The main focus is on Eulerian structure and stability, with most examples existing outside of the parameter space we expect for ocean eddies. More relevant to the ocean is early work on cases with strong background rotation and with a focus on stratification (e.g. Barcilon \& Pedlosky 1967) or instability (e.g. Hart $\&$ Kittelman 1996). Small values of the aspect ratio $H / R$ and consequences for the sidewall layers have also been explored in numerous studies (e.g. Pedlosky \& Spall 2005). Works that explore Lagrangian aspects include Fountain et al. (2000) and Lackey \& Sotiropoulos (2006), both of which concentrate on cases with very high Rossby number and low Reynolds number.

Our attention is restricted to steady flow, and we find that instability is avoided if the Reynolds number $R_{e}=U R / v=\delta \Omega R^{2} / v$ is limited to values less than $\sim 5000$. Also, we concentrate on cases in which the aspect ratio $H / R$ is $O(1)$, characteristic of deep convection cells in the ocean, but also explore values down to $1 / 50$, which would be appropriate for many submesoscale eddies. Our model is highly idealized, but this is desirable given the rich variety of regimes that arise. The dimensionless parameters of primary importance are $R_{o}, E$ and the forcing amplitude as defined below. The Reynolds number is related to the above by

$$
R_{o}=E R_{e}(H / R)^{2} .
$$

\subsection{Fully nonlinear numerical model}

We conduct numerical simulations using Nek5000, which solves the Navier-Stokes equations based on the spectral element method (Patera 1984; Maday \& Patera 1989; Fischer 1997). Nek5000 has been developed by P. F. Fischer and colleagues (see http: //nek5000.mcs.anl.gov/index.php/Main_Page). Previous studies within oceanographic context conducted with Nek5000 include simulations of gravity currents (Özgökmen et al. 2007; Özgökmen, Iliescu \& Fischer 2009a,b) and upper-ocean mixed layer instabilities (Özgökmen et al. 2011; Özgökmen \& Fischer 2012; Özgökmen et al. 2012). Nek5000 is configured to integrate the equations

$$
\left(\frac{\partial}{\partial t}+\boldsymbol{u} \cdot \nabla\right) \boldsymbol{u}=R_{o}^{-1} 2 \boldsymbol{k} \times \boldsymbol{u}-\nabla p+R_{e}^{-1} \nabla^{2} \boldsymbol{u}
$$

and

$$
\nabla \cdot \boldsymbol{u}=0 .
$$

All variables are now non-dimensional, with lengths scaled by $R$, time by $1 / \delta \Omega$, and velocities by $\delta \Omega R$ (see appendix A). 
Spectral element methods combine the geometric flexibility of finite elements with the numerical accuracy of spectral expansions. The cylindrical domain $(1 \geqslant r \geqslant 0,1 \geqslant$ $z \geqslant 0)$ is discretized using 640 elements. In almost all experiments presented below, solutions on each element are represented by Legendre polynomials of order $N=21$, leading to 5990923 grid points. The model is integrated using a time step of $10^{-3}$ for a total integration period changing from 10 to 200 until a full steady state is reached, depending primarily on the value of $E$. Most integrations have been carried out on 128 processors of the recent Linux cluster, while we have also run higher resolution experiments with $N=39(38,183,887$ grid points) on 320 processors of a Cray SE6m system to check for numerical convergence.

At the upper lid, the horizontal velocity imposed in the model is given by

$$
\begin{aligned}
& u(x, y, 1)=-4 y(1-r) \\
& v(x, y, 1)=4\left(x-x_{o}\right)(1-r) .
\end{aligned}
$$

For $x_{o}=0$, the lid velocity is purely azimuthal and equivalent to the non-dimensional version of (2.3). The velocity vanishes at the outer edge $r=1$ regardless of the value of $x_{o}$. The flow produced is steady, has $\partial / \partial \theta=0$, and is referred to as the 'undisturbed' flow. A disturbed flow is produced through finite $x_{o}$, which places the zero of the lid speed at $y=0$ and $x=x_{o}$. This effectively creates a low azimuthal mode structure. The parameter $x_{o}$ is therefore a measure of the strength of the disturbance.

\subsection{Phenomenological (kinematic) model}

We will occasionally employ a kinematic model for purposes of illustration and, more importantly, to verify tendencies predicted by our resonance width calculation. The latter would be quite difficult using the full Navier-Stokes equations. Let $(u, v, w)$ represent radial, azimuthal and vertical velocity components. Then

$$
u=\frac{(2 z-1)}{3} r(a-r), \quad v=\alpha r \quad \text { and } \quad w=z(1-z)\left(\frac{2}{3} a-r\right)
$$

describes a steady, incompressible velocity field satisfying the no-normal-flow conditions along the cylinder boundaries $(z=0,1$ and $r=a)$. This azimuthally uniform velocity field consists of a horizontal swirling motion with constant vorticity $2 \alpha$. There is also an overturning circulation with radial velocity $u$ that is inward in the bottom half of the cylinder $(0<z<1 / 2)$ and outward in the top half $(1 / 2<z<1)$, and a vertical velocity $w$ that is positive for $2 a / 3>r \geqslant 0$ and negative for $r>2 a / 3$.

Each trajectory in this field lives on a torus, a property that can be verified from the streamfunction

$$
\psi(r, z)=\frac{1}{3} r^{2}(r-a) z(z-1),
$$

for the overturning circulation: $u=-(1 / r) \partial \psi / \partial z$ and $w=(1 / r) \partial \psi / \partial r$. Level curves of $\psi$ form closed contours in the $(r, z)$ plane and these represent the slices through tori on which trajectories live. All trajectories are integrable and each maintains a fixed value of $\psi$ as it winds about the torus, as illustrated in the next section.

In their laboratory and numerical study of (mostly) non-rotating flow, Fountain et al. (2000) induce chaos by tilting an impellor that drives the flow from the top. Here we impose a disturbance that also has azimuthal structure, but zero vertical velocity. The disturbance is specified in Cartesian $(x, y, z)$ coordinates, which allow a finite velocity at the origin $r=\left(x^{2}+y^{2}\right)^{1 / 2}=0$. The horizontal perturbation velocity is specified 
through the streamfunction

$$
\tilde{\psi}(x, y ; z, t)=\frac{1}{2}\left(a^{2}-r^{2}\right)\left[\varepsilon_{1}\left(x-x_{o}\right)+\varepsilon_{2} r^{2} \cos \left(n_{\theta} \theta\right)\right](1-\beta z),
$$

with $\tilde{u}^{(x)}=-\partial \tilde{\psi} / \partial y, \tilde{v}^{(y)}=\partial \tilde{\psi} / \partial x$, and $\theta=\arctan (y / x)$. The disturbance can destroy the axial symmetry by adding an offset factor $x_{o}$ relative to the centre $(x=y=r=0)$. It can also introduce vertical shear through the parameter $\beta$ and a stationary azimuthal wave with wavenumber $n_{\theta}$. The strengths of these various components are controlled through amplitude parameters $\alpha, \varepsilon_{1}, \varepsilon_{2}, \beta$ and $x_{o}$. Additional details are given in appendix B.

The kinematic model should not be viewed as a substitute for the Navier-Stokes simulations. It does not, for example, have boundary layers, so detailed comparisons would be meaningless. It is used simply to make illustrations of objects that are generic given the background geometry and to implement verification of trends that are independent of dynamics.

\section{Theory for steady perturbations of azimuthally symmetric, steady states}

In an undisturbed $(\partial / \partial \theta=0)$ state, trajectories spiral up through the interior of the tank and into the top Ekman layer, where they spiral outward (figure 1). They spiral downward around the outer edge and inward at the bottom. Regardless of the model, each trajectory remains on a torus as it executes an overturning cycle. Examples taken from the velocity field (2.8) are constructed by tracing six colourcoded trajectories over many overturning cycles (figure $2 a$ ). The red trajectory ascends near the centreline of the tank and descends near the outer walls. It is quasiperiodic and so traces out a slightly different path over each overturning cycle, eventually covering a torus. All other trajectories on this surface are identical but for a phase shift in $\theta$. If the red and yellow tori are pealed away (figure $2 b$ ), a periodic (green) orbit is revealed. This trajectory also lives on a torus but the surface is no longer revealed by a single trajectory. Inside lie other tori containing quasiperiodic or periodic orbits (figure $2 c$ ), and at the centre lies a single, periodic orbit (figure $2 d$ ).

A vertical slice through the objects shown in figure $2(a)$ reveals the tori in section (figure 3). This view is akin to a Poincare section in that the trajectories are marked with a dot each time they cross the fixed vertical section. For a quasiperiodic trajectory, the dots will densely cover a closed curve that forms the intersection with the corresponding torus. For periodic trajectories, such as the green one, there will be a finite number of intersections points. For example the green trajectory in figure $2(b)$ intersects each side of the figure 3 plot four times.

There are two mechanisms by which perturbations to this azimuthally uniform background state can generate chaotic motion. The first involves the resonant breakup of tori, and this is discussed in $\$ \S 3.1-3.3$ below. The second mechanism involves the breakup of the central streamline at $r=0$ into stable and unstable manifolds, as discussed in $\S 3.4$.

\subsection{Action-angle-angle coordinates}

The trajectories of the undisturbed state are all regular (non-chaotic). At question is the survival, under perturbation, of the tori on which they live. Does a particular torus simply deform under perturbation, continuing to exist as a two-dimensional material surface, or does it break up? The answer depends on whether the motion on particular torus is resonant, in a Lagrangian sense. In order to identify the condition for resonance, one needs to define frequencies of motion in the azimuthal and meridional 
(a)

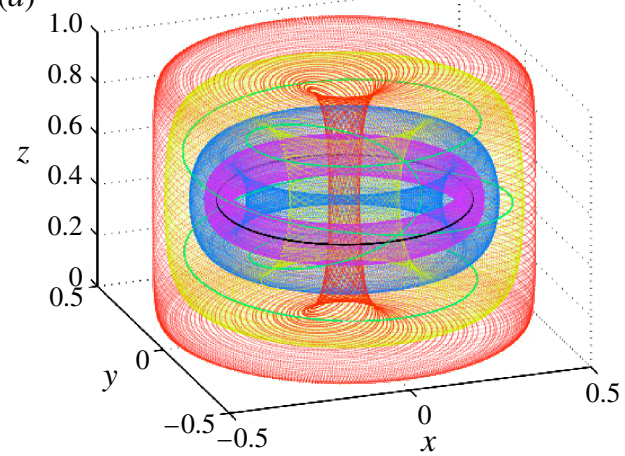

(c)

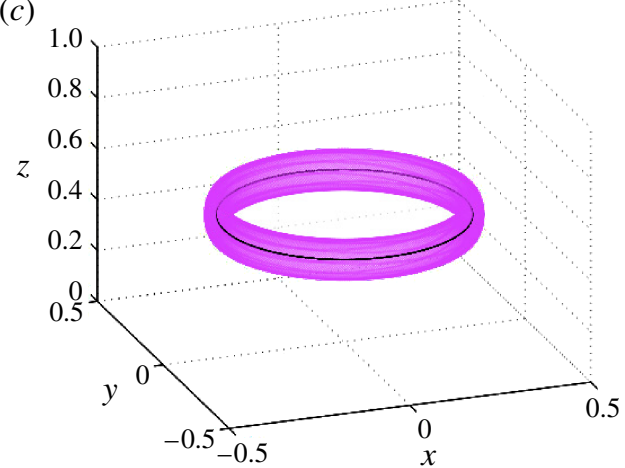

(b)

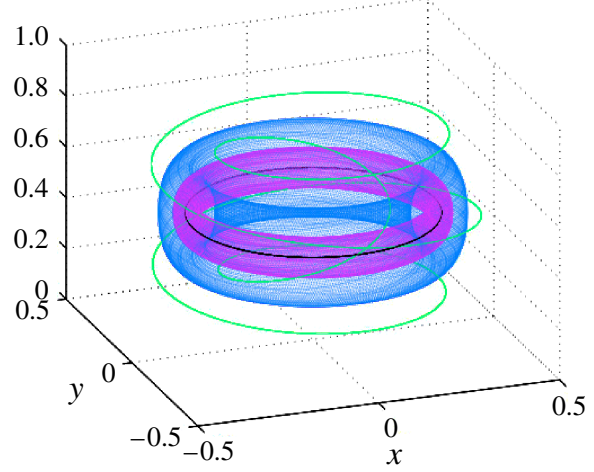

(d)

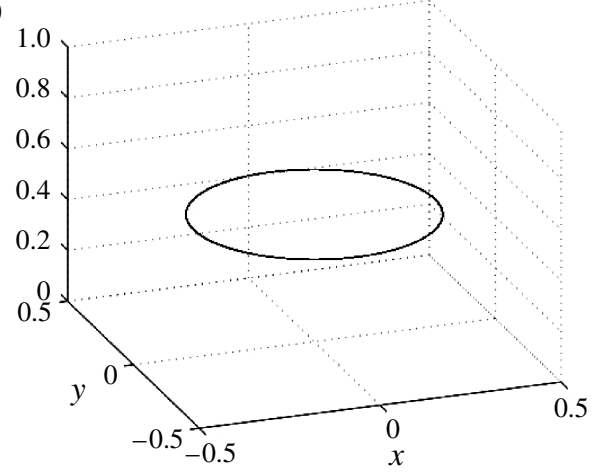

FIGURE 2. Trajectories and associated tori for the steady, axisymmetric state given by (2.8), with $a=0.5, \alpha=0.35$. The full surfaces are associated with quasiperiodic trajectories. $(a)$ The surfaces traced out by six trajectories with different colours. $(b)$ The two outermost tori are taken away, revealing a periodic (green) orbit. $(c, d)$ More surfaces are pealed away, revealing smaller tori (purple) and a limiting periodic trajectory (black).

(overturning) directions. It is not immediately obvious that one can do so, since the period between successive trajectory crossings of the vertical plane (figure 3) generally varies with each crossing. Mezic \& Wiggins (1994) show that a consistent period can be defined for a given torus if the crossings are measured in a transformed coordinate system: so-called 'action-angle-angle' variables. Certainly the geometry of the nested tori suggest a natural coordinate system in which each torus is assigned a label $I$ (the 'action'), and the position on that torus is prescribed by two angles, as suggested in figure 4. One may define the angles in a variety of ways, but Mezic \& Wiggins (1994) identify a particular set (see below) for which all trajectories with given $I$ not only have well defined frequencies but also have constant angular velocities.

The transformations, which are detailed in appendix $\mathrm{C}$, describe the formulation of a transverse angle $\phi$ that increases linearly from zero to $2 \pi$ following a trajectory that winds around the shaft of the torus. In the azimuthal direction, the new coordinate $\tilde{\theta}$ is similar to the standard azimuthal angle $\theta$, but has an origin that is tilted relative to $\theta$, as suggested in figure 4 . Successive crossing of this origin by a trajectory occur at uniform time intervals $T_{\theta}$. The equations for motion along an unperturbed torus are 


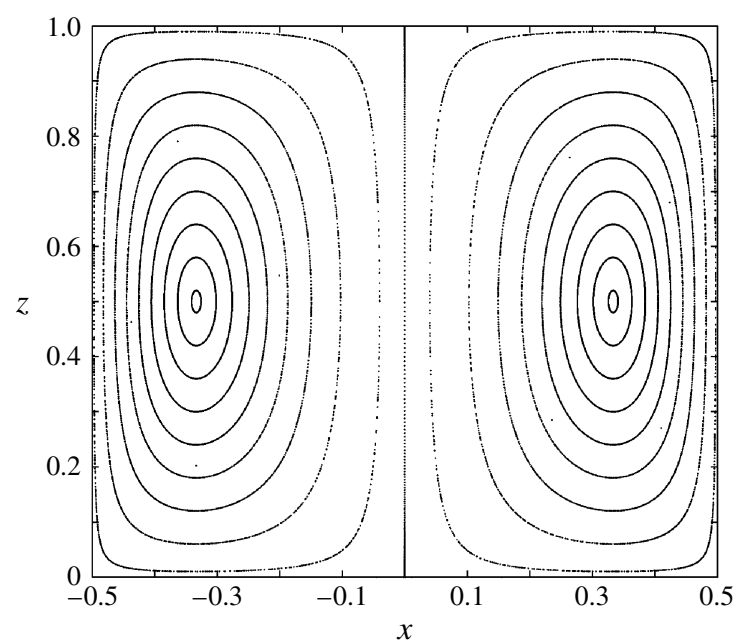

FIGURE 3. The tori and periodic orbit of figure 2 as seen in a Poincaré section taken in the $(y, z)$ plane.

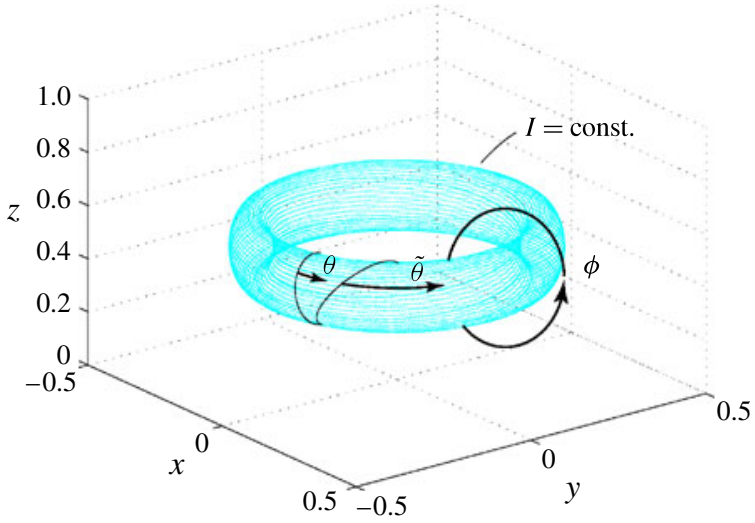

Figure 4. (Colour online) Action-angle-angle variables $(\phi, \tilde{\theta}, I)$ for the undisturbed flow. The standard azimuthal angle $\theta$ is measured relative to the $x$-axis. The angle $\tilde{\theta}$ is measured relative to a shifted reference curve that depends on $\phi$, as shown.

thus

$$
\frac{\mathrm{d} \phi}{\mathrm{d} t}=\Omega_{\phi}(I)=\frac{2 \pi}{T_{\phi}(I)}, \quad \frac{\mathrm{d} \tilde{\theta}}{\mathrm{d} t}=\Omega_{\theta}(I)=\frac{2 \pi}{T_{\theta}(I)} \quad \text { and } \quad \frac{\mathrm{d} I}{\mathrm{~d} t}=0
$$

where $T_{\theta}(I)=2 \pi / \Omega_{\theta}(I)$ and $T_{\phi}(I)=2 \pi / \Omega_{\phi}(I)$ are the azimuthal and meridional periods of motion. In order to measure $T_{\theta}(I)$ on a particular torus, one must locate the contour $\tilde{\theta}=0$ and this is generally non-trivial. Fountain et al. (2000) estimated $T_{\theta}(I)$ by averaging over many zero crossings of $\theta=0$. 


\subsection{Periodic orbits and barrier destruction}

Periodic orbits cycle through the same path and therefore sample any steady perturbation in a periodic manner. The expected resonance is analogous to that occurring in two-dimensional, steady systems forced by a time-periodic disturbance. For small perturbations, resonance leads to the breakup of the torus along with neighbouring tori, and to the generation of a corresponding band of chaos. As shown by Mezic \& Wiggins (1994), and reviewed by Fountain et al. (2000), the transformed variable set allows invocation of an extension of the KAM theorem and the conclusion that some non-resonant tori survive a small perturbation. We will advance this subject a bit more by calculating the resonance width.

Following Fountain et al. (2000), suppose that on a particular torus $I$ the azimuthal and meridional periods obey

$$
\frac{T_{\theta}}{T_{\phi}}=\frac{m}{n}
$$

for some integers $m$ and $n$. Then the trajectories on that torus are periodic, with period $n T^{(\theta)}=m T^{(\phi)}$. Under perturbation, any such torus will break along with those nearby in the manner described in $\S 3.3$. For tori with irrational $T_{\theta} / T_{\phi}$ the trajectories are quasiperiodic. As applied to this problem, the KAM theorem guarantees that at least some tori with sufficiently irrational $T_{\theta} / T_{\phi}$ (i.e. those satisfying a Diophantine condition written down by Fountain et al. (2000)) will survive. This condition is not sufficient and it is impossible to say whether any individual torus will survive. The only guarantee is that the majority of these tori survive, so one is assured that the weakly perturbed flow will contain some barriers to chaotic transport.

The green trajectory shown in figure $2(b)$ lives on a torus covered with similar periodic trajectories. As shown in figure 3, each trajectory crosses the vertical halfslice $(0 \leqslant x \leqslant 0.5,0 \leqslant z \leqslant 1)$ four times during each complete circuit, and thus $n=4$. (Strictly speaking, one should count trajectory crossings in the tilted plane corresponding to $\tilde{\theta}=0$, but in the example presented the count is the same as in a vertical plane.) Similarly, each trajectory crosses each horizontal slice within its range 2 time, so $m=2$. Figure 5 shows the result of the resonant breakup of this torus for a disturbance with $\varepsilon_{1}=0.15, \alpha=0.35, x_{o}=-0.25, \beta=1$ and $\varepsilon_{2}=0$. Note the braided region of chaotic behaviour, dark and cloudy in figure 5 , in the vicinity of the original resonant torus. Other tori, one coloured blue in the figure, have survived the perturbation. In a three-dimensional view (figure 6), this blue torus is just a slightly distorted donut.

A fascinating aspect of a resonance is that, along with chaos and enhanced stirring, it also produces new invariant tori. These are shown in figure 5 as 'islands' of nested contours, one coloured green, within the resonant layer. The expected number of islands in the vertical plane is $n$, whereas a horizontal Poincaré section is expected to show $m$ islands. At the centre of the islands is an elliptical fixed point corresponding to a periodic orbit. This orbit is encased in tori that resemble twisted hula hoops, as illustrated by the green object in figure 6 . When sliced vertically, the twisted torus appears as the green contours of figure 5. Elsewhere in figure 5 lie other islands, including an outer chain of 5 that appear as clear regions imbedded in the main cloudy region. These result from the breakup of a torus with $n=5$. Between this and the $n=4$ chain lies a less distinct chain with $n=9$. 


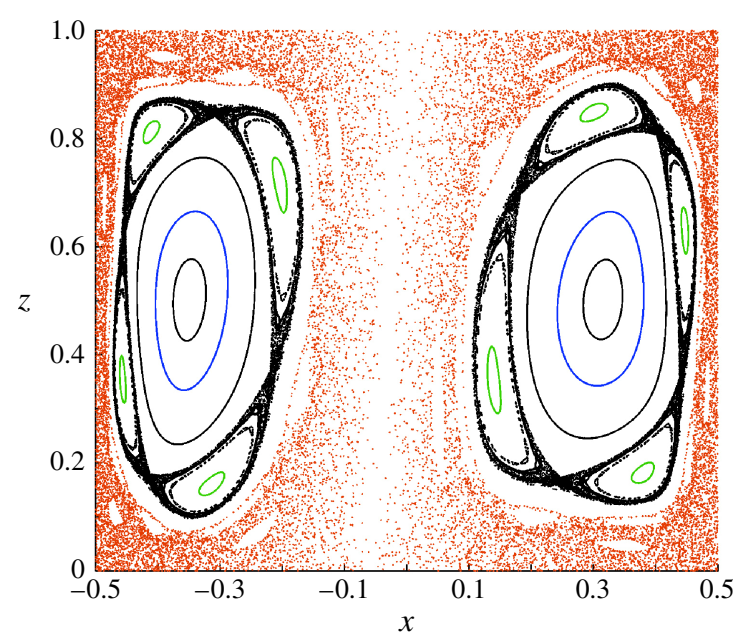

FIGURE 5. A Poincaré section based on a perturbation of the figure 3 flow with $a=0.5$, $\varepsilon_{1}=0.15, \varepsilon_{2}=0, \alpha=0.35, x_{o}=-0.25$ and $\beta=1$ in $(2.10)$.

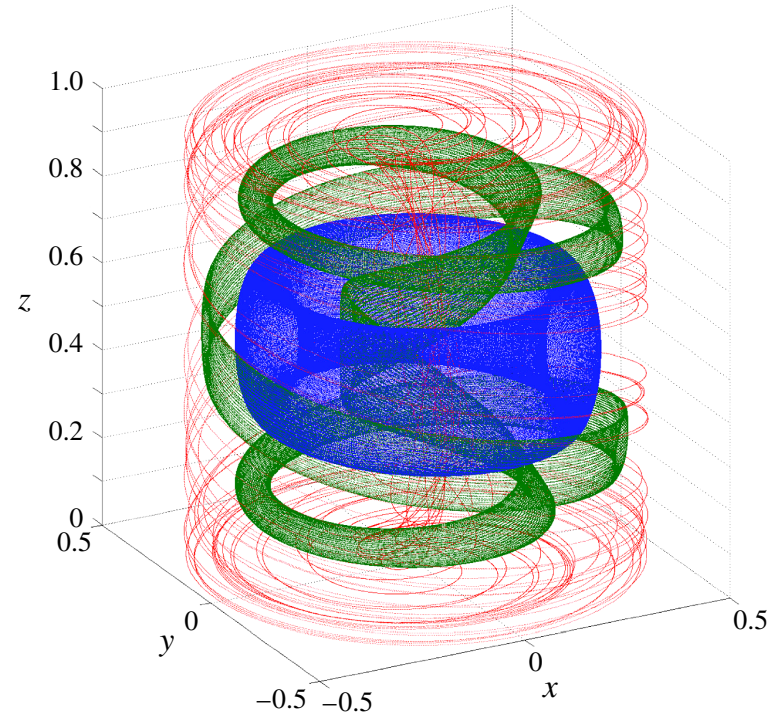

FIGURE 6. Examples of the material tori in the disturbed flow of figure 5. The blue rings of figure 5 are sections of the blue torus shown here. The green island rings in figure 5 correspond to the torus with $n=4$, shown here in green. The red trajectory inhabits the large chaotic region.

\subsection{Resonant width}

The extent of chaos about a resonant tori depends on how well the structure of the perturbation projects onto the structure of the resident periodic trajectories, and on how nearly resonant the nearby tori are. These properties can be formalized through a calculation of the width of a resonant layer, an exercise that also reveals the existence and geometry of the island structures. Resonant width calculations have been done for 
two-dimensional flows with time-periodic perturbations (see Lichtenberg \& Lieberman (1992) for examples). Here we work through the steady three-dimensional case. By standard definition, the resonance width is the half-width of the islands that appear in the resonant layer, as measured in the change $\Delta I$ in the action variable from the centre to the outer edge of the island. Thus, for a resonant torus $I=I_{o}$, tori in the range $I_{o}-\Delta I<I_{o}<I_{o}+\Delta I$ will break up.

Consider the trajectory equations for the perturbed flow in action-angle-angle variables:

$$
\begin{aligned}
\dot{I} & =\varepsilon F^{0}(I, \phi, \tilde{\theta}), \\
\dot{\phi} & =\Omega_{\phi}(I)+\varepsilon F^{1}(I, \phi, \tilde{\theta}), \\
\dot{\tilde{\theta}} & =\Omega_{\theta}(I)+\varepsilon F^{2}(I, \phi, \tilde{\theta}) .
\end{aligned}
$$

The dot denotes a time derivative and the perturbation amplitude $\varepsilon$ is assumed $\ll 1$.

Owing to periodicity in the angle variables, it is possible to expand the disturbance functions in Fourier series, for example

$$
F^{0}(I, \phi, \tilde{\theta})=\sum_{m, n=-\infty}^{\infty} F_{n m}^{0}(I) \sin \left(n \phi-m \tilde{\theta}+\alpha_{n m}\right),
$$

where $\alpha_{n m}$ are phases. The evolution of $I$ along a trajectory is then given by

$$
\dot{I}=\varepsilon \sum_{m, n=-\infty}^{\infty} F_{n m}^{0}(I) \sin \left(n \phi-m \tilde{\theta}+\alpha_{n m}\right) .
$$

For flow in the vicinity of a particular torus $I=I_{o}$, the phase function in (3.4) can be approximated as

$$
\begin{aligned}
\eta(t) & =n \phi(t)-m \tilde{\theta}(t)+\alpha_{n m} \\
& =n \phi(0)-m \tilde{\theta}(0)+\alpha_{n m}+t\left[n \Omega_{\phi}\left(I_{o}\right)-m \Omega_{\theta}\left(I_{o}\right)\right]+O(\delta I)+O(\varepsilon)
\end{aligned}
$$

which follows from integration of (3.3b) and (3.3c). Thus, for non-resonant tori $\left(n \Omega_{\phi}\left(I_{o}\right) \neq m \Omega_{\theta}\left(I_{o}\right)\right)$ the argument increases linearly with time, the right-hand side of (3.5) is sinusoidal, and the displacement $\delta I=I-I_{o}$ of a trajectory from the reference torus varies periodically in $\theta$ and $\phi$ but does not grow. However, if $I_{o}$ is resonant, $n \Omega_{\phi}\left(I_{o}\right)=m \Omega_{\theta}\left(I_{o}\right)$, then the coefficient of $t$ in the phase function is replaced by

$$
n \Omega_{\phi}(I)-m \Omega_{\theta}(I)=\left.n \frac{\mathrm{d} \Omega_{\phi}}{\mathrm{d} I}\right|_{I_{o}} \delta I-\left.m \frac{\mathrm{d} \Omega_{\theta}}{\mathrm{d} I}\right|_{I_{o}} \delta I+\cdots
$$

so faster growth in $\delta I$ is possible.

Temporarily disregarding the non-resonant terms, equation (3.5) can now be approximated as

$$
\dot{\delta} I=\varepsilon F_{n m}^{0}\left(I_{o}\right) \sin (\eta)
$$

and

$$
\dot{\eta}=\left[\left.n \frac{\mathrm{d} \Omega_{\phi}}{\mathrm{d} I}\right|_{I_{o}}-\left.m \frac{\mathrm{d} \Omega_{\theta}}{\mathrm{d} I}\right|_{I_{o}}\right] \delta I,
$$




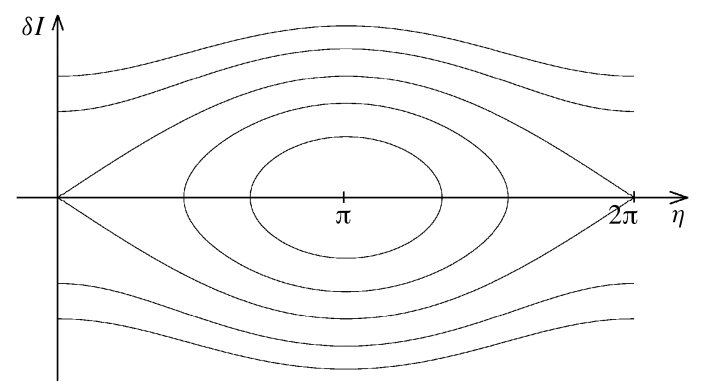

FIGURE 7. Phase space portrait for flow near resonant torus. The contours are of $H(I, \eta)=$ constant for the function defined in (3.11).

which can be expressed in the Hamiltonian form

$$
\dot{\delta I}=-\partial H / \partial \eta \quad \text { and } \quad \dot{\eta}=\partial H / \partial(\delta I)
$$

where

$$
H=\varepsilon F_{n m}^{o}\left(I_{o}\right) \cos \eta+\left[\left.n \frac{\mathrm{d} \Omega_{\phi}}{\mathrm{d} I}\right|_{I_{o}}-\left.m \frac{\mathrm{d} \Omega_{\theta}}{\mathrm{d} I}\right|_{I_{o}}\right] \frac{\delta I^{2}}{2} .
$$

Each trajectory maintains a fixed value of $H$ and so the level sets of $H(I, \eta)$ correspond to invariant surfaces in physical space. In the phase space $(I, \eta)$ (figure 7) the contours of constant $H$ consist of closed curves (corresponding to islands) and open contours (corresponding to perturbed, but intact tori). The closed curves are contained within a heteroclinic separatrix. Chaos arises when the non-resonant terms are resurrected, leading to breakup of the separatrix.

In order to visually transform the invariant surfaces $H=$ constant back to physical space, consider the phase variable $\eta(\phi(t), \tilde{\theta}(t))$ evaluated at $t=0$ :

$$
\eta(\phi(0), \tilde{\theta}(0))=n \phi(0)-m \tilde{\theta}(0)+\alpha_{n m} .
$$

Here $\phi(0)$ and $\tilde{\theta}(0)$ represent the initial position of a trajectory, but we can also view (3.12) from an Eulerian perspective by tracing the changes in $\eta$ when $\tilde{\theta}(0)=0$ and $\phi(0)$ is varied through $2 \pi$. This traces a path that travels one cycle about a tilted closed curve similar to that shown in figure 4. (If one sets $\delta(I)=0$, then the path is exactly the closed curve shown.) Over this circuit, (3.12) shows that $\eta$ changes by $2 n \pi$, and so if the phase portrait has one island in each $2 \pi$ interval, as in figure 7 , there will be $n$ islands in the tilted Poincaré section taken at constant $\tilde{\theta}$.

The separatrix between the closed and open contours corresponds to the contour $H_{s e p}=\varepsilon F_{n m}^{o}$. The maximum excursion of this contour, which corresponds to the halfwidth of the trapped region and thus defines the resonance width, is

$$
\Delta I=2 \sqrt{\frac{\varepsilon F_{n m}^{0}\left(I_{o}\right)}{\left|n\left(\mathrm{~d} \Omega_{\phi} / \mathrm{d} I\right)\right|_{I_{o}}-\left.m\left(\mathrm{~d} \Omega_{\theta} / \mathrm{d} I\right)\right|_{I_{o}} \mid}}=2 \sqrt{\frac{\varepsilon F_{n m}^{0}\left(I_{o}\right)}{\left|n \Omega_{\phi}\left(I_{o}\right)\left(\mathrm{d} \ln \left(\Omega_{\phi} / \Omega_{\theta}\right) / \mathrm{d} I\right)\right|_{I_{o}} \mid}},
$$

where the second equality follows from use of the resonance condition (3.7). It can be seen that the resonant response is $O\left(\varepsilon^{1 / 2}\right)$ whereas the non-resonant terms would have led to a $O(\varepsilon)$ response had they been included. 
The term $F_{n m}^{0}$ measures the projection of the spatial structure of the disturbance onto the periodic orbits, whereas the derivative of $\ln \left(\Omega_{\phi} / \Omega_{\theta}\right)$ indicate the proximity of resonance of neighbouring tori. Large resonant widths are encouraged by a close structural match, small $n$ (as seen in the denominator of the final expression) and gradual variation of $\Omega_{\phi} / \Omega_{\theta}$ between neighbouring tori. If the derivative of $\Omega_{\phi} / \Omega_{\theta}$ vanishes for some resonant $I$, then higher-order terms in the expansion (3.7) must be considered.

Evaluation of (3.13) for a particular torus is fiendishly difficult: one must first compute the action-angle-angle variables and then somehow Fourier expand the disturbance in them. However, the simplicity of the kinematic model makes it possible to verify at least some of the general tendencies seen in (3.13). As described in appendix D (also see figure 21), we have checked the square-root dependence on the disturbance amplitude $\varepsilon$ as well as the factor $\mathrm{d} \Omega_{\phi} / \mathrm{d} I$ and found very good agreement in both cases.

\subsection{Stable and unstable manifolds}

A second mechanism for the generation of chaos is the breakup of the central streamline that connects the top and bottom of the cylinder at $r=0$ in the undisturbed state (figure 3). The analogue of this object in two dimensions would be the streamline that separates two horizontal gyres or eddies of opposite sign. In the two-dimensional case, a time-dependent perturbation generally causes this boundary to split into distinct material contours, the stable and unstable manifolds. The contours generally intersect each other in multiple locations and the resulting 'turnstile' lobes contain fluid that is being exchanged between the two gyres. Samelson \& Wiggins (2006) present a thorough description of this process.

The present situation is different, partly because the central streamline no longer separates two distinct regions: it simply lies at the centre of a swirling flow. Under a steady perturbation the streamline splits into two manifolds, one emanating from the lower-boundary, parabolic stagnation point and one from the upper-boundary, parabolic stagnation point. Using methodology developed by Rypina et al. (2011) we have constructed the stable manifold of the perturbed flow (figure 8). It is a curve that contains all material that approaches a stagnation point on the upper boundary asymptotically in time. An unstable manifold emanates from the lower boundary. It is not presented here but Fountain et al. (2000, figure 16) show a laboratory visualization from a dye release. These curves do not appear to intersect, but verification of this property is difficult. It is apparent, however, that the curves wander about and fill a finite subvolume of the cylinder. Fluid trajectories in this subvolume are subject to initial condition sensitivity since each must lie arbitrarily close to the stable manifold and must eventually pass close to the hyperbolic region at the top of the cylinder. We therefore expect chaotic behaviour in the volume filled by the manifolds, and this volume includes the central axis and outer edges of the cylinder, as exemplified in figure 5 .

\section{Chaos and stirring in the Navier-Stokes flow}

Visualizations of invariant tori, including islands, by dye release are presented by Fountain et al. (2000). The parameter range and forcing mechanisms are not broadly applicable for ocean eddies, so we have used the numerical model to map out a more relevant parameter space, roughly $10^{-4} \leqslant E \leqslant 1$ and $0.2 \leqslant R_{o} \leqslant 1$. Although the fundamental geometry of the underlying Lagrangian structures remains the same, the 


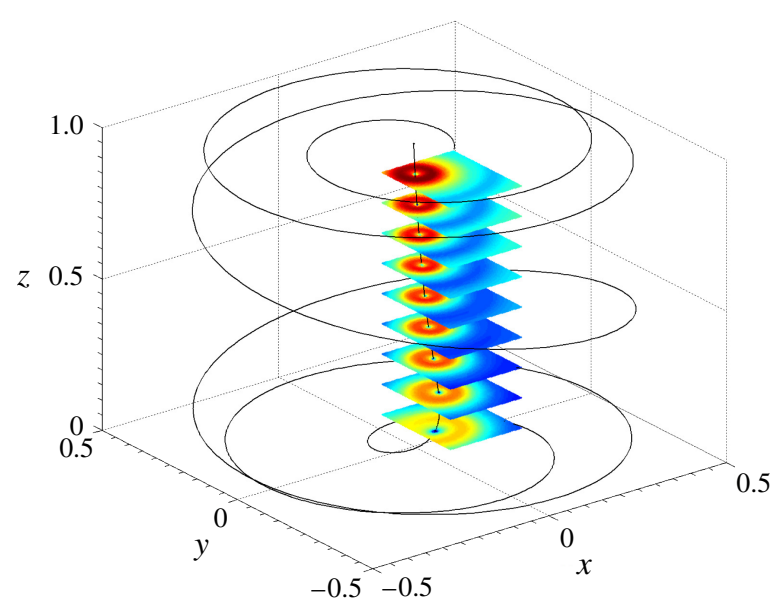

FIgURE 8. (Colour online) The stable manifold of the flow shown in figure 5. This curve contains fluid that approaches a stagnation point at the top lid asymptotically in time. The manifold was produced using the arclength-based complexity method described by Rypina et al. (2011). Select horizontal slices of the arclength field near the centre of the cylinder are shown in colour. Blue indicates smaller arclength, red indicates larger arclength.

qualitative pictures that emerge, particularly for small Ekman number, can be quite different than anything found in previous work.

\subsection{Poincaré sections versus finite-time Lyapunov exponents}

A representative result from the numerical model, with parameter settings $x_{0}=-0.02$, $E=1 / 20, R_{o}=1$ and $H / R=1$ (and therefore $R_{e}=20$ ), is shown in figure 9 . Figure $9(a)$ contains a Poincaré section in the $(x, z)$ plane; figure $9(b)$ shows finite-time Lyapunov exponents (FTLEs) over the same space. The details of the computation of FTLEs from the nonlinear model is described by Özgökmen et al. (2012). For any location in the vertical slice shown, the FTLE is the maximum exponential rate of separation between a fluid trajectory beginning at that location and trajectories beginning at nearby locations (both in and out of plane). The expectation is that, for finite but large integration time, chaotic regions will be characterized by much larger FTLE values than regular regions. The results are sensitive to the time $T$ over which the trajectories are integrated, and the distinction between the regular and chaotic zones becomes more apparent at longer integration times.

The case shown contains invariant tori that have survived perturbation along with a complicated assortment of islands produced by resonances at various $n$. There is good correspondence between the tori and islands in the upper panel and low FTLE regions in the lower panel. The broad chaotic region that occupies the cylinder centre and outer edges is represented by uniformly high (although grainy) FTLE distribution. In order to achieve correspondence between the fine structures seen in the Poincaré sections and FTLE fields, and integration of time $T$ of several thousand dimensionless time units, or $T / 2 \pi$ differential rotation cycles, was generally found necessary.

\subsection{Lagrangian regimes for weak disturbances}

We now consider a sequence of runs (figure 10) with variable Ekman number $E$, with Rossby number fixed at $R_{o}=0.2$ or $R_{o}=1.0$ (left and right columns), and with 


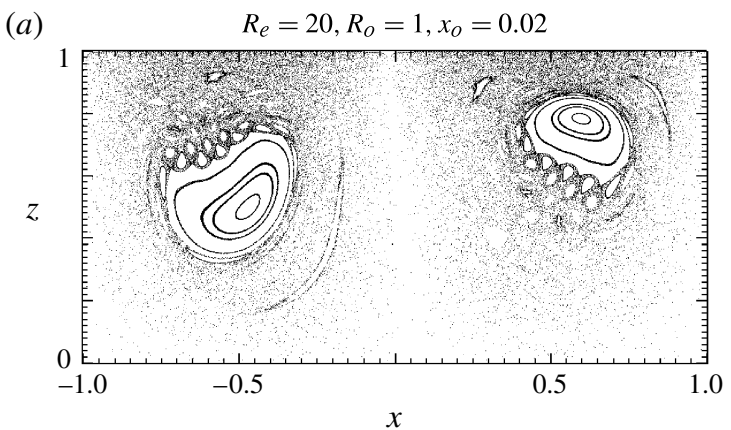

(b)

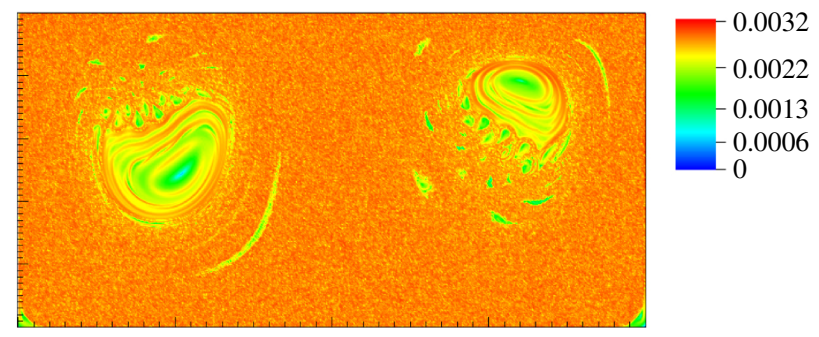

FIGURE 9. (Colour online) Numerical solution for $R_{o}=1, E=1 / 20, H / R=1$ and $x_{o}=-0.04$ (so that $R_{e}=20$ ). (a) A Poincaré section over a vertical slice along the $x$-axis. The trajectory that forms the broad chaotic (cloudy) regions was tracked for 5500 azimuthal cycles. (b) FTLEs over the same $(x, z)$ plane.

fixed forcing amplitude $x_{o}=-0.02$. For $O(1)$ values of $E$, as in frames $(a, b)$, the top and bottom Ekman layers strongly overlap and viscous effects are felt throughout the entire depth. The tori are very stable, although magnification reveals the presence of island chains that are very thin, have very high $n$, and thus have the appearance of dashed curves. Chaos is thus confined to very thin resonant layers sandwiched between invariant tori. We will refer collectively to cases with $E=O(1)$ as regime I: they are characterized by viscous effects extending top to bottom and thin resonant layers.

As $E$ is lowered below unity (frames $c-j$ ) the outer tori break up and trajectories through the central axis and outer edges of the tank become dominantly chaotic. Resonances with lower $n$ appear within the inner tori and these result in island chains with $n=4(e), n=3(g)$ and $n=1(i, j)$, all with larger widths. The cases with $n=1$ are interesting because the island is quite large and possesses a homoclinic geometry that differs from the braided (heteroclinic) geometry characteristic of islands with $n>1$. A good example can be seen in frame $(i)$, with the centre of the island in the left half of the image near $x=-0.4$ and $z=0.5$. This should be distinguished from the nest of closed contours centred at $x=-0.6$ and $z=0.65$, which correspond the innermost tori of the undisturbed flow. The island is produced not from the breakup of the $n=1$ periodic trajectory lying at the centre of the nest $(I=0)$, but rather from a resonant torus lying some distance from the centre (i.e. $I>0$ ), and also with $n=1$. The two islands shown near the centre of the nested tori in frame $f$ are connected and we have verified that the periodic orbit at the centre of the one island also travels through the centre of the other. These 'regime II' cases are characterized by moderately small $E$, large and homogeneous regions of chaos through the central and outer portions of the cylinder, thick resonant layers with low $n$, and weak barrier stability. 
(a)

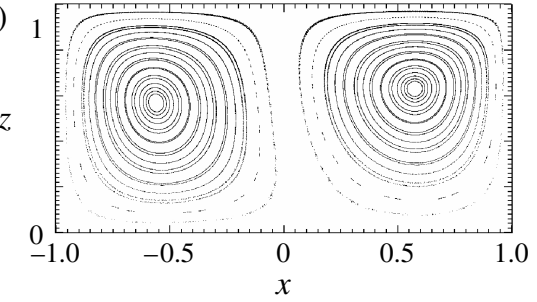

(c)

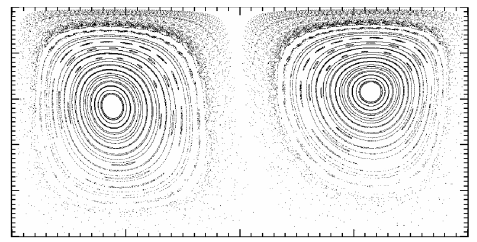

(e)

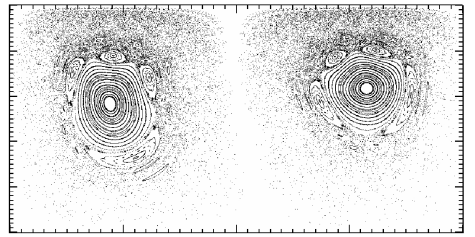

(g)

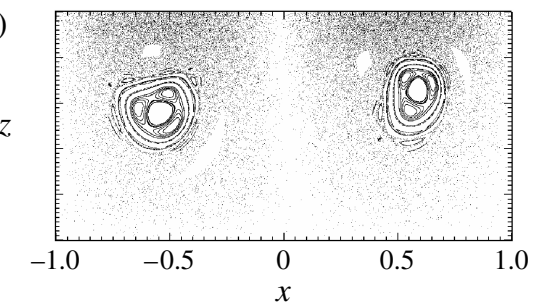

(i)

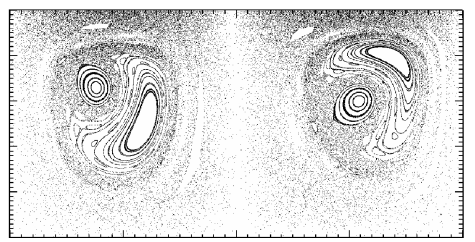

(k)

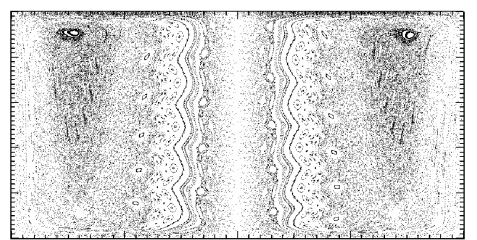

(b)

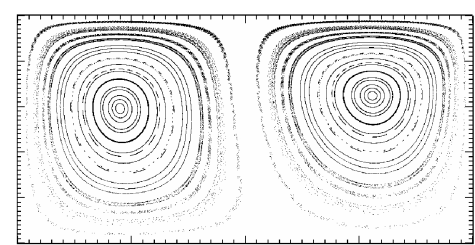

(d)

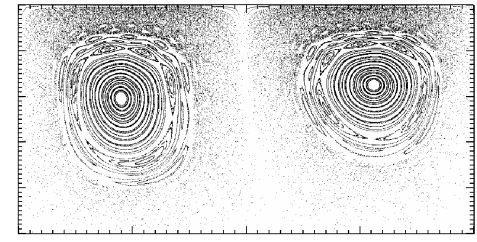

(f)

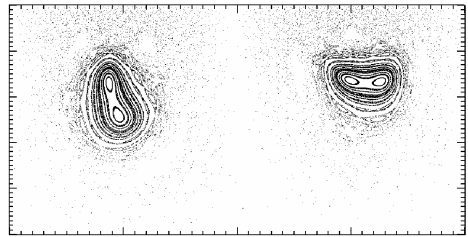

(h)

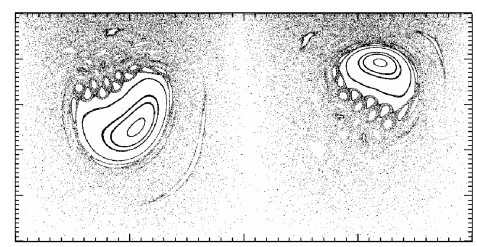

(j)

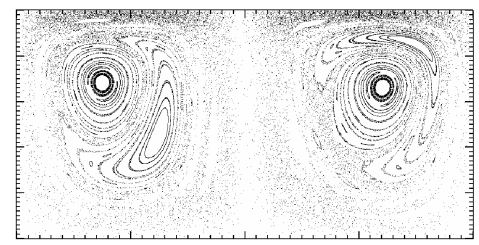

(l)

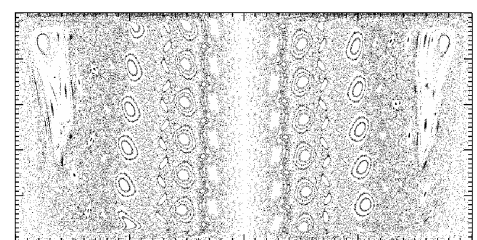

FIGURE 10. Poincaré sections in the $(r, z)$ plane based on numerical solutions, all with forcing amplitude $x_{o}=-0.02$. The left column has $R_{o}=0.2$ and the right column has $R_{o}=1$, and $E$ decreases downward through the rows as labelled: $(a) E=1, R_{o}=0.2\left(R_{e}=0.2\right) ;(b)$ $E=1, R_{o}=1\left(R_{e}=1\right) ;(c) E=1 / 4, R_{o}=0.2\left(R_{e}=0.8\right) ;(d) E=1 / 4, R_{o}=1\left(R_{e}=4\right) ;(e)$ $E=1 / 8, R_{o}=0.2\left(R_{e}=1.6\right) ;(f) E=1 / 8, R_{o}=1\left(R_{e}=8\right) ;(g) E=1 / 20, R_{o}=0.2\left(R_{e}=4\right)$; (h) $E=1 / 20, R_{o}=1\left(R_{e}=20\right)$; ( $\left.i\right) E=1 / 50, R_{o}=0.2\left(R_{e}=10\right)$; (j) $E=1 / 50, R_{o}=1\left(R_{e}=\right.$ 50); (k) $E=1 / 2000, R_{o}=0.2\left(R_{e}=400\right)$; (l) $E=1 / 2000, R_{o}=1.0\left(R_{e}=2000\right)$. 
If $E$ is lowered to $1 / 2000$ (frames $k-l$ ) the Ekman layers become well separated and the interior region becomes increasingly rigid in the vertical. This behaviour is consistent with the Taylor-Proudman theorem as discussed below. The centre of the undisturbed tori, which must have positive radial velocity above and negative velocity below, can therefore not lie in the rigid interior. Resonances again are dominated by very high $n$, an aspect that will also be explored in the next section. These 'regime III' flows have $E \leqslant 10^{-3}$, thin boundary layers, and semirigid interiors with thin resonant layers and high $n$ values.

\subsection{Trajectories, islands and resonant layers}

Much of the variation of behaviour exhibited in figure 10 can be explained through consideration of the structure of periodic trajectories, as reflected by typical $m$ and $n$ values. According to the asymptotic theory for small $E$ (see (A 6)-(A 8) of appendix A) the vertical velocity in the geostrophic interior is $O\left(E^{1 / 2}\right)$ while the azimuthal velocity is $O(1)$. (The dimensional interior azimuthal and vertical velocities approach fixed values as the tank deepens. The dimensional overturning period therefore grows while the azimuthal period remains fixed.) For $E \ll 1$ fluid parcels cycle azimuthally many times as they slowly ascend or descend through the cylinder. The radial velocity in the upper and lower Ekman layers is $O(1)$ and the overturning period is therefore set by the $O\left(E^{1 / 2}\right)$ vertical velocity. For an undisturbed torus $I$, the ratio of azimuthal period $T_{\theta}(I)$ to overturning period $T_{\phi}(I)$ is then $O\left(E^{1 / 2}\right)$. A undisturbed torus with periodic orbits has

$$
\frac{T_{\theta}}{T_{\phi}}=\frac{m}{n}=O\left(E^{1 / 2}\right) .
$$

Resonant island chains in the vertical plane are therefore associated with large $n$ for very small $E^{1 / 2}$, whereas smaller $n$ becomes possible as $E$ grows.

The above trend reflects the behaviour shown in figure 10 as $E$ increases from $1 / 2000$ to $1 / 50$. However, when $E$ is further increased the trend reverses and the lowest $n$ value for visible islands again increases. At $E=1 / 100$ examples of periodic trajectories can be found with low $n$, as exemplified by the example with $n=3$ shown in figure 11(a). At $E=1$ all trajectories have high $n$, as exemplified by a case shown in figure $11(b)$. The asymptotic theory also becomes formally invalid as $E$ approaches $O(1)$, so guidance from this quarter may no longer be trustworthy. In physical terms the Ekman layers begin to overlap and the top and bottom boundaries come into viscous contact with each other. Intuition suggests that this should suppress the vertical velocity, thereby increasing $T_{\phi}(I)$ and making $n$ larger. This prediction is supported by a calculation (end of appendix A) of the vertical velocity for arbitrary $E$, but using infinite cylinder radius and rigid lid forcing to simplify. The resulting vertical velocity is independent of $r$ and is given at the mid-depth $(z=1 / 2)$ by

$w\left(r, \frac{1}{2}\right)=\frac{E^{1 / 2}}{2} \frac{\left(\sinh \left(1 / 2 E^{1 / 2}\right)-\sin \left(1 / 2 E^{1 / 2}\right)\right)\left(\cosh \left(1 / 2 E^{1 / 2}\right)-\cos \left(1 / 2 E^{1 / 2}\right)\right)}{\sin ^{2}\left(1 / 2 E^{1 / 2}\right) \cosh ^{2}\left(1 / 2 E^{1 / 2}\right)+\cos ^{2}\left(1 / 2 E^{1 / 2}\right) \sinh ^{2}\left(1 / 2 E^{1 / 2}\right)}$.

A plot of this function (figure 12) over the range $0 \leqslant E^{1 / 2} \leqslant 1$ shows that $w$ indeed increases from zero in proportion to $E^{1 / 2}$, but that a maximum is reached at $E^{1 / 2}=0.2218$, above which $w$ decreases continuously. The small circles show the locations of experiments for $R_{o}=0.2$ and $x_{o}=-0.02$, and each is labelled with the value of the smallest observed $n$ based a count of islands within chains. Although the 
(a)

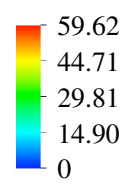

(b)

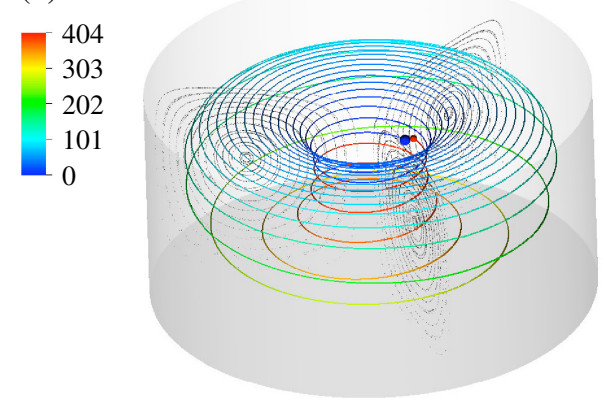

FIgURE 11. (Colour online) Examples of periodic trajectories with $(a) T_{\theta} / T_{\phi}=1 / 3$ and (b) $T_{\theta} / T_{\phi} \ll 1$. Here $R_{o}=1$ for both cases and $(a) E=1 / 100$ and $(b) E=1$.

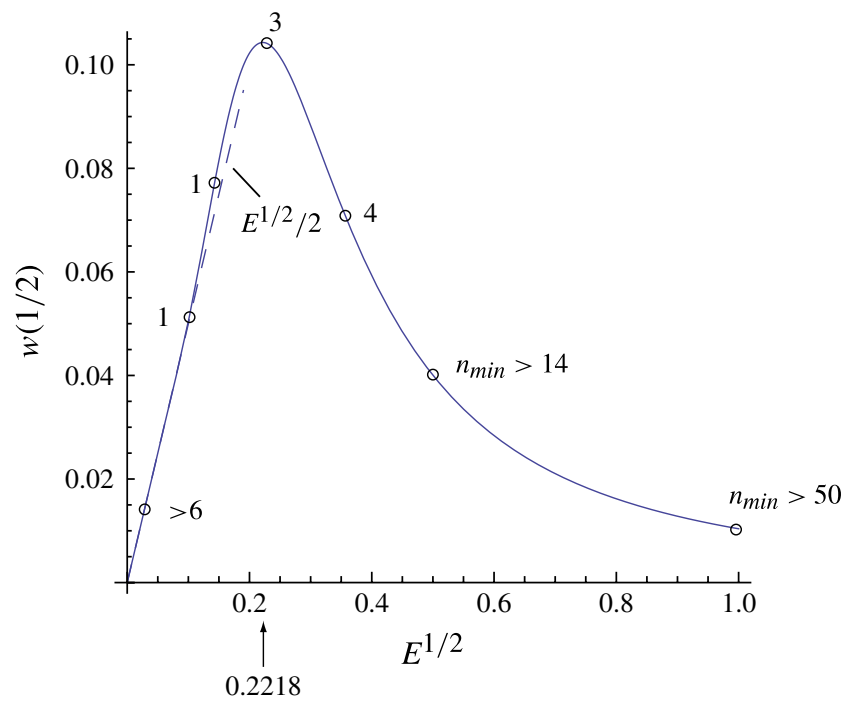

FIGURE 12. (Colour online) Graph of the mid-depth value of $w$ versus $E^{1 / 2}$ (see (4.2)) for the case $R \rightarrow \infty$ and rigid lid rotation. Dots mark values of $E=1 / 2000,1 / 100$, $1 / 50,1 / 20,1 / 8,1 / 4$ and 1 and the minimum observed value of $n$ for island chains for corresponding runs with $R_{o}=0.2$ and $x_{o}=-0.02$ is indicated next to each dot.

maximum in $w$ does not exactly correspond to the minimum in $n$ it is clear that higher values of $w$ generally correspond to lower $n$.

Further support for this reasoning comes from direct calculation of the periods $T_{\theta}$ and $T_{\phi}$ for various tori in the undisturbed flow and for various values of $E$. (Since the action-angle-angle coordinates are unknown, $T_{\theta}$ is estimated as an average of periods of successive zero crossings of $\theta=0$ over many cycles.) To identify the various concentric tori, we first locate the position in the $(r, z)$ of the central elliptic point. Then keeping $z$ constant we decrease $r$ to zero in increments, thereby sampling through inner to outer tori, and $T_{\phi}$ and $T_{\theta}$ are found for each case. Figure 13 shows the distribution of $T_{\theta} / T_{\phi}$ for the cases $E=1,1 / 100$ and $1 / 2000$, and it is clear 


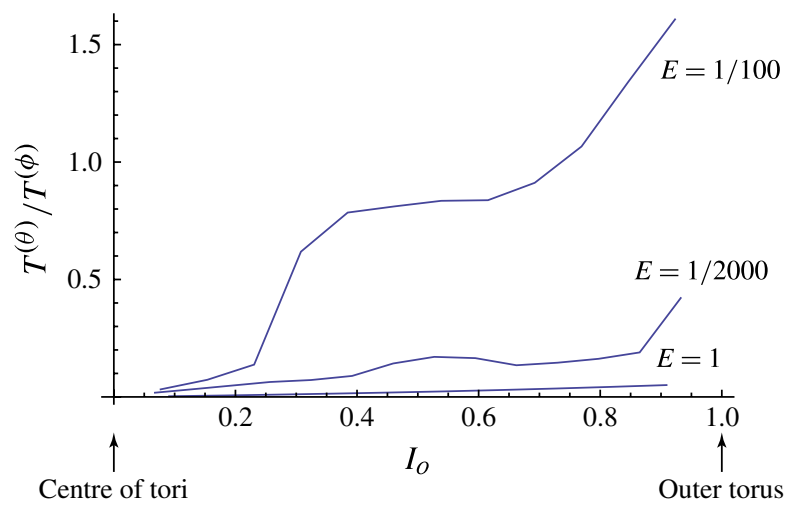

FIGURE 13. (Colour online) Estimates of the ratio $T_{\theta} / T_{\phi}$ of azimuthal to overturning periods over tori of the undisturbed states with $R_{0}=1$ and for three values of $E$. Trajectories are started along a constant $z$ line that begins at the elliptical centre $\left(r_{o}, z_{o}\right)$ of the tori and extends to $r=0$. Here $I_{o}$ is an action coordinate, equal to $\left(r_{o}-r\right) / r_{o}$. The estimates do not include tori at the very centre (small $I_{o}$ ), or tori spanning the outer edge and central axis of the cylinder $\left(I_{o} \approx 1\right)$.

that $O(1)$ values (and therefore small $n$ values) occur mainly within the intermediate case $E=1 / 100$.

The widths of the resonant layers are generally largest in the intermediate regime (II). Here $T_{\phi}$ and $T_{\theta}$ are of similar magnitudes for at least some of the periodic orbits and the corresponding trajectories have low $n$. The perturbation itself always has a simple structure, with a low-mode azimuthal structure imposed at the top lid and a vertical structure that is constrained by Ekman layer dynamics to spiral, decay and be nearly constant in the geostrophic interior. The low values of $n$ contribute to a small denominator in (3.13) and the projection factor $F_{n, m}^{0}$ is presumably large (although extremely difficult to calculate). In contrast, values of $n$ are much larger in regimes (I) and (III) leading to smaller resonance widths. The contribution to $\Delta I$ from the derivative of $\ln \left(\Omega_{\phi} / \Omega_{\theta}\right)$ (i.e. of $\ln \left(T_{\theta} / T_{\phi}\right)$ ) is harder to assess. Figure 13 suggests that this derivative is generally smaller in regimes (I) and (III), but is also small over an intermediate range of tori in regime (II).

\subsection{Eulerian flow}

The presence of islands in Poincaré sections should not be interpreted as an indication of small overturning cells in the Eulerian flow. In fact, additional overturning cells do not arise in any of the flows analysed. As examples, figure 14 shows the Eulerian overturning circulations for the flow fields used to produce the Poincaré sections of figure $10(a, i, k)$ above. Note that each vector field contains just a single overturning cell. The reader may also wish to reflect on the striking similarity between the Eulerian velocity field for the cases $E=1$ and $E=1 / 50$ (figure 14a,b), along with the equally striking differences in the corresponding Poincaré sections (figure 10a,i).

\section{Analysis}

\subsection{Implications of the Taylor-Proudman theorem}

The KAM theorem and the resonance width formula place constraints on Lagrangian behaviour of prescribed vector fields that are restricted only by the condition of 

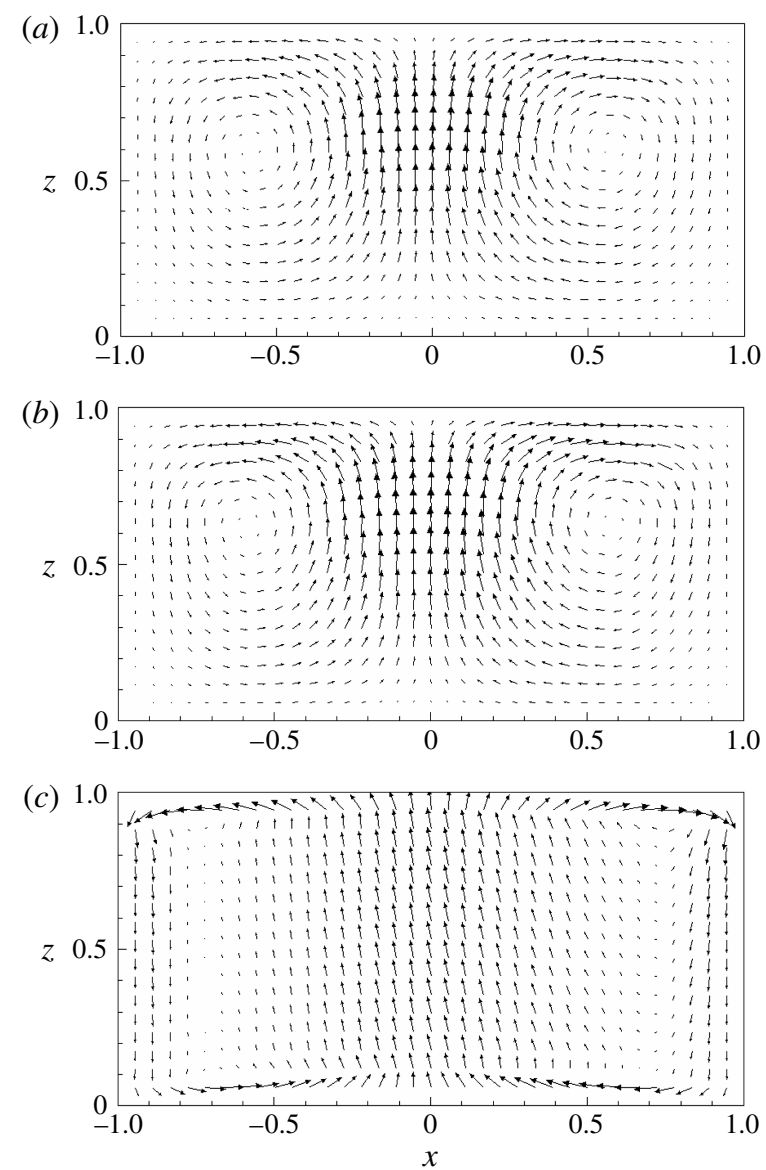

FIGURE 14. The overturning circulation as shown by vectors consisting of the radial and vertical velocity components along the vertical plane $y=0$. All cases have $R_{o}=0.2$ and $x_{o}=-0.02$, with $(a) E=1$, (b) $E=1 / 50$ and (c) $E=1 / 2000$. The sections correspond to figure $10(a, i, k)$.

incompressibility. In principle, further constraints should follow from considerations involving momentum and thermodynamic laws. In the present situation, one such constraint follows from the Taylor-Proudman theorem, which requires that for steady, inviscid, homogeneous flow with $R_{o} \ll 1$, all three velocity components must be independent of $z$. The continuity equation then implies that the horizontal velocity is divergence free, so a streamfunction $\psi(x, y, z)$ exists for the horizontal velocity at each $z$, and

$$
u(x, y)=-\frac{\partial \psi}{\partial y} \quad \text { and } \quad v(x, y)=\frac{\partial \psi}{\partial x} .
$$

The $z$-dependent part of $\psi$ must take the form $\psi(x, y, z)=\psi_{o}(x, y)+g(z)$, so the horizontal gradient of $\psi$ must be independent of $z$ and each streamline must remain aligned with one directly above. The flow may have finite vertical velocity but fluid trajectories must remain on vertically aligned sheets. This property does not require 
radial symmetry and therefore applies to the disturbed flow. If contours of constant $\psi$ are closed, the sheets are topologically equivalent to cylinders.

Ideally, the cylinders should extend from the top of the bottom Ekman layer to the bottom of the top Ekman layer. They would also lie away from the thickest sidewall Stewardson layers (of width $O\left(E^{1 / 4}\right)$ ), although these layers are very weak in the present simulations. A trajectory may leave one cylinder by entering an Ekman layer, and then re-enter the interior along another cylinder. It may not switch cylinders within the inviscid interior. If the flow is driven by a rigid, rotating lid, upward moving trajectories enter the top Ekman layer and are carried downward through the Stewardson layers into the bottom Ekman layer, from which they re-enter the interior. For the forcing used in the present experiments, trajectories leave and re-enter the interior through the same Ekman layer. Regardless of the forcing, nearly all trajectories must pass through a viscous layer. Exceptions include those trajectories lying near the radius that separates the upwelling and downwelling regions, where $w$ is small.

These conditions are never strictly met in the numerical simulations due to the finite value of $R_{o}$ and perhaps due to viscous influence from the sidewalls, but strong vertical rigidity exists in the interior in cases considered with $E=1 / 2000$ (e.g. figure $14 c$ ). The presence of resonant layers and islands in the corresponding Poincaré section (figure 10k) is not inconsistent with this property. As a trajectory spirals upwards, it passes through the section at the same horizontal position, but a different vertical position, at each crossing. This is true whether the trajectory lies on an undisturbed torus, or on one of the twisted hula hoops that form as a chain of island in the vertical section. After the trajectory overturns and spirals upwards again, it may cross the section at a different horizontal position, remaining on the same hula hoop. In this way, the island chain is eventually traced out.

One might expect that the rate of chaotic stirring (quantified below) will be small for $E \ll 1$. The barrier stability evident in figure $10(a, b)$ suggests the same for $E=O(1)$. The intermediate regimes should have the largest stirring rates and this is verified below. The prediction of non-monotonic stirring has also been put forth by Mezic (2001) in connection with contained, three-dimensional steady flows. The central hypothesis is that the domain contains an inviscid interior region in which fluid parcels preserve the Bernoulli function $B=p / \rho+|\boldsymbol{u}|^{2} / 2+g z$. If the gradient of $B$ is non-zero in the interior, trajectories are constrained to move along surfaces of uniform $B$. Chaotic stirring is thus limited to the viscous boundary layers, whose thickness is $O\left(R_{e}{ }^{-1 / 2}\right)$, and the prediction is that the stirring rate should also decrease as $R_{e}{ }^{-1 / 2}$ where $R_{e}$ is large but below the threshold of turbulence. Stirring is also predicted to be small within the highly viscous range $R_{e} \ll 1$. The predictions of non-monotonic stirring and of $R_{e}{ }^{-1 / 2}$ decay are verified in the experiments of Lackey $\&$ Sotiropoulos (2006) for the case of counter-rotating top and bottom (but with no background rotation).

In our rotating cylinder flow, the strictly inviscid conditions envisioned by Mezic (2001) hold for few, if any, trajectories. In addition, there are now two nondimensional parameters, $E$ and $R_{o}$, and not just $R_{e}$. Despite these differences, the vertical sheets required by the Taylor-Proudman theorem bear some similarity to the closed, invariant surfaces envisioned by Mezic (2001), and it is natural to ask whether the stirring rate is similarly constrained.

\subsection{Bulk stirring rates}

In order to quantify changes in the stirring rate over a range of $E$ and $R_{o}$ we track the evolution of a passive tracer with concentration $C(x, y, z, t)$ and having small 
(a)

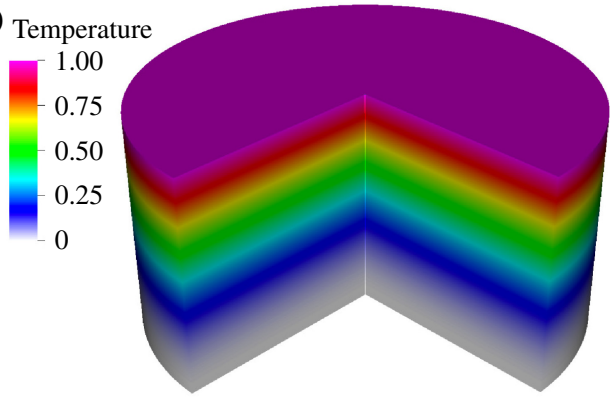

(c)

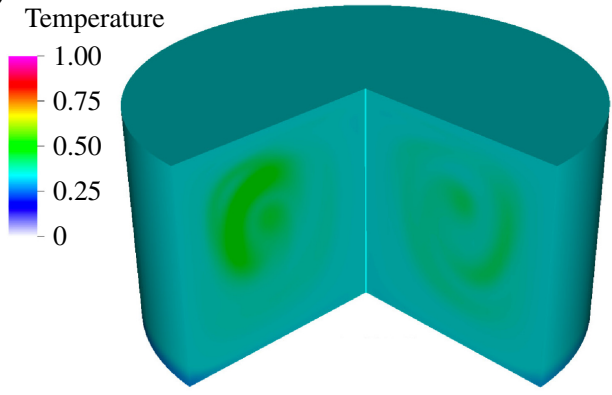

(b)

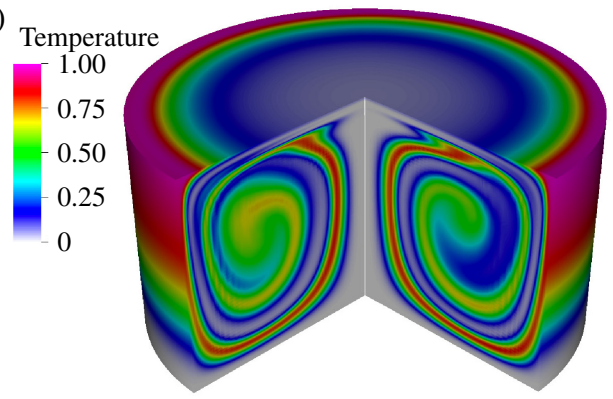

(d)

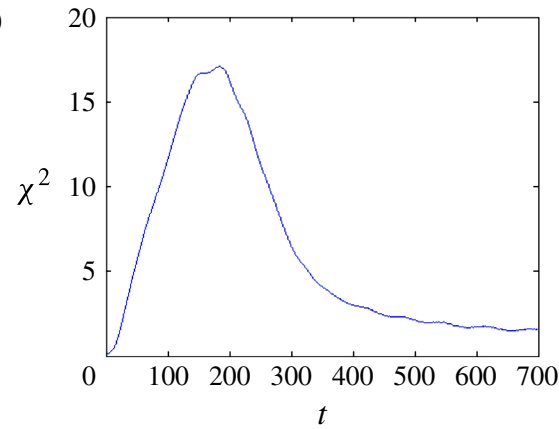

Figure 15. (Colour online) The evolution of a passive tracer, with $E=1 / 50, R_{o}=0.2$, $x_{o}=-0.02$, shown in $(a)$ at $t=0$, in $(b)$ at $t=30$, near the beginning of the conservative stirring stage, and in $(c)$ at $t=300$, during the mixing stage. The time history of the tracer variance function $\chi^{2}(t)$, as defined in (5.2), is shown in $(d)$.

dimensionless diffusivity $\kappa\left(=10^{-6}\right)$. The initial tracer concentration is as shown in figure $15(a)$. The tracer variance function

$$
\chi^{2}(t)=\int_{V}|\nabla C|^{2} \mathrm{~d} v / \int_{V}|C|^{2} \mathrm{~d} v
$$

can be used to quantify the stirring rate (Pattanayak 2001). Typical of all numerical runs, the tracer undergoes an initial stage dominated by conservative filamentation (figure $15 b$ ) during which $\chi^{2}(t)$ increases linearly with time (e.g. figure $15 d$ ). After a finite time, amplification of the tracer gradients as a result of stretching and folding is arrested by diffusion and $\chi^{2}(t)$ reaches a peak value. Thereafter $\chi^{2}(t)$ is eroded by diffusion and decays, corresponding to a mixing stage (figure $15 c$ ). The average slope of the $\chi^{2}(t)$ curve during the initial, nearly linear growth stage is a bulk measure of the stirring rate and is independent of $\kappa$, provided that the latter is kept sufficiently small. In measuring this average slope, we have verified that shape exhibits the nearly linear increase to a peak from case to case, and we have spot checked to verify that the slope is independent of $\kappa$. We have also verified that the numerator in (5.2) remains nearly constant throughout time, so that the variations of $\chi^{2}(t)$ are contained in the numerator.

We first consider the change in stirring rate as a function of Ekman number for fixed $R_{o}$ and small disturbance amplitude $x_{o}$. Measurements from the Navier-Stokes simulation for $x_{o}=-0.02$ and $x_{o}=-0.08$ are represented by blue and purple dots in figure $16(a)$, plotted as a function of $E^{-1 / 2}$, and all for $R_{o}=0.2$. Shallow (deep) 

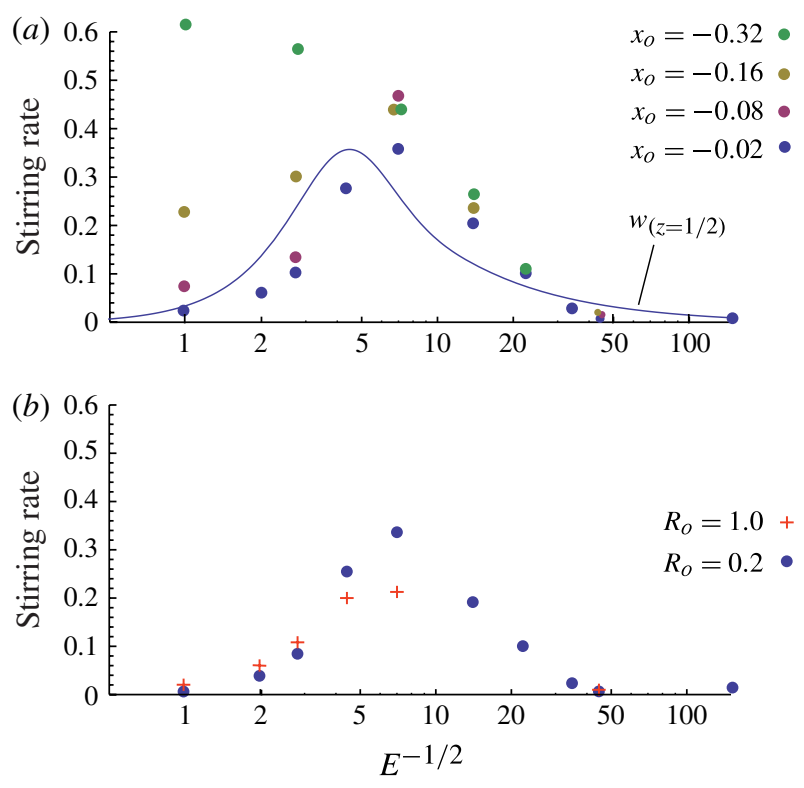

FIGURE 16. Stirring rates calculated from the Navier-Stokes model. The stirring rate is defined as the average value of $\mathrm{d} \chi^{2}(t) / \mathrm{d} t$ over the initial period of conservative tracer filamentation, for which the growth in $\chi^{2}(t)$ is nearly linear. The results are plotted as a function of $E^{-1 / 2}$, so that deep eddies lie to the right and shallow eddies to the left. In $(a)$ the Rossby number is fixed at $R_{o}=0.2$ and the forcing amplitude is increased. In $(b)$ the forcing amplitude is fixed at $x_{o}=-0.02$ and the Rossby number is increased from 0.2 to 1.0. The continuous curve in $(a)$ shows the vertical velocity at mid-depth for an unbounded cylinder: the same function plotted in figure 12 but in terms of $E^{-1 / 2}$ and scaled so that its maximum value equals that of the data for $x_{o}=-0.02$.

eddies lie to the left (right) on the horizontal axis. For both sets of data, the stirring rate is non-monotonic, with relatively low values for $E^{-1 / 2}=O(1)$ and for $E^{-1 / 2} \gg 1$, and with a peak at $E^{-1 / 2} \cong 7$. The latter is proximal to the peak in the plot of vertical velocity given by (5.2), which is shown by the blue curve and has a maximum at $E^{-1 / 2} \cong 4.5$. So far, all seems to be consistent with the visual evidence provided by the figure 10 sections for small $x_{o}$ (which suggest maximum stirring at intermediate $E^{-1 / 2}$ ), with the resonance condition (3.2) (which, along with (5.2), suggest resonances with low $n$ at intermediate $E^{-1 / 2}$ ), with the resonance width formula (3.13) (which suggests thicker resonant layers for low $n$ ), and with the Taylor-Proudman theorem (which suggests low stirring for large $E^{-1 / 2}$ ). We note, however, that the measured stirring rate for large $E$ does not decay in proportion to $E^{1 / 2}$, which by (2.4) would be equivalent to the $R_{e}^{-1 / 2}$ decay predicted by Mezic (2001). This mismatch can be seen by comparing the blue curve with the data points for $E^{-1 / 2} \gg 1$. The former decays in proportion to $E^{1 / 2}$ whereas the later indicate a more rapid decay.

As the perturbation amplitude is increased, the stirring rates grow. For $E^{-1 / 2}$ greater than 30-40, where the Taylor-Proudman theorem is most relevant, this growth is minimal. However, for $E^{-1 / 2}=O(1)$, the growth is surprisingly large. As shown by the gold and green dots representing $x_{o}=-0.16$ and $x_{o}=-0.32$ in figure $16(a)$, this trend eventually destroys the non-monotonic stirring trend, at least over the range of $E$ considered. Stirring is now maximal for shallow eddies. Surprisingly, Poincaré sections 
(a)

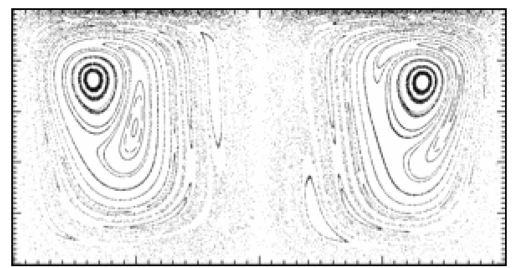

(c)

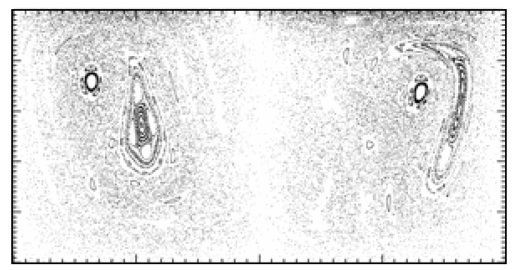

(b)

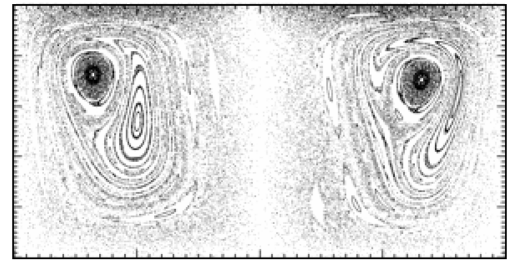

(d)

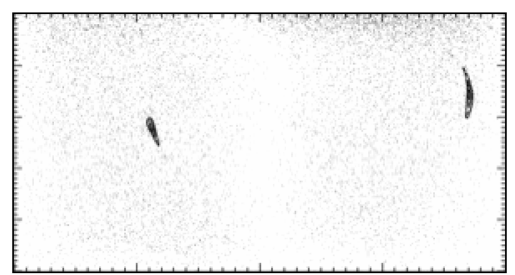

FIGURE 17. Poincaré sections in the $(r, z)$ plane based on numerical solutions, all with $R_{o}=1, E=1 / 100$ and with increasing magnitude of the forcing amplitude $x_{o}$, as labelled: $(a)$ $x_{o}=-0.02 ;(b) x_{o}=-0.04 ;(c) x_{o}=-0.08 ;(d) x_{o}=-0.16$.

for these last two cases (not shown) are similar to those in figure $10(a, b)$ in that the domain is filled with thin resonant layers sandwiched between barriers. Plots of the velocity field confirm that the flow has not split into multiple gyres.

Attempts to interpret these results raises the question of what $\chi^{2}(t)$ is actually measuring. Filamentation will occur as the result of shear alone, without Lagrangian chaos, so increases in tracer variance involves some combination of chaotic motion and shear. A benchmark for shear alone is provided by the undisturbed flows, which have no chaotic orbits. The stirring rates are typically an order of magnitude below what is measured for the cases that are weakly or moderately perturbed and therefore have similar Eulerian structure. This suggests that the increased stirring rates for small disturbances are largely due to chaos. For large disturbances this conclusion requires additional support; at least this is suggested by those cases with $E=1$. We have not pursued this question further.

Also apparent in figure $16(a)$ is that for intermediate values of $E^{-1 / 2}$ the stirring rate ceases to grow, and in a few cases slightly decreases, as $x_{o}$ increases. This saturation roughly coincides with the forcing threshold beyond which most barriers are destroyed, as documented below.

When the Rossby number is increased from 0.2 to 1.0 , the stirring rates at fixed values of $x_{o}$ undergo modest changes, as shown in figure 16(b). For the moderately small $E^{-1 / 2}$ the stirring rate actually decreases.

\subsection{The distribution of chaos; global transport}

The bulk stirring gives a measure of the average rate of stretching and folding but yields no information about the distribution of chaos. As the magnitude of the forcing parameter $x_{o}$ increases, resonant layers increase in width and begin to overlap (after Zaslavsky \& Chirikov 1972; Chirikov 1979), destroying the separating barriers. In addition, the chaotic region occupied by the stable and unstable manifolds emanating from the cylinder top and bottom expands. Eventually all global barriers are destroyed and trajectories are free to roam nearly the entire volume. Small regular islands often persist, but they occupy a small fraction of the volume. Rapid stirring due to chaos is widespread and almost uniform. An example is shown in figure 17, where we 
(a)$$
\text { (a) }
$$

Regular, possibly with very thin resonant layers

Substantial regions of chaos and or regular

Mostly chaotic but some large barriers remain $\odot$ Only tiny regular islands

All chaotic

(2) Inconclusive
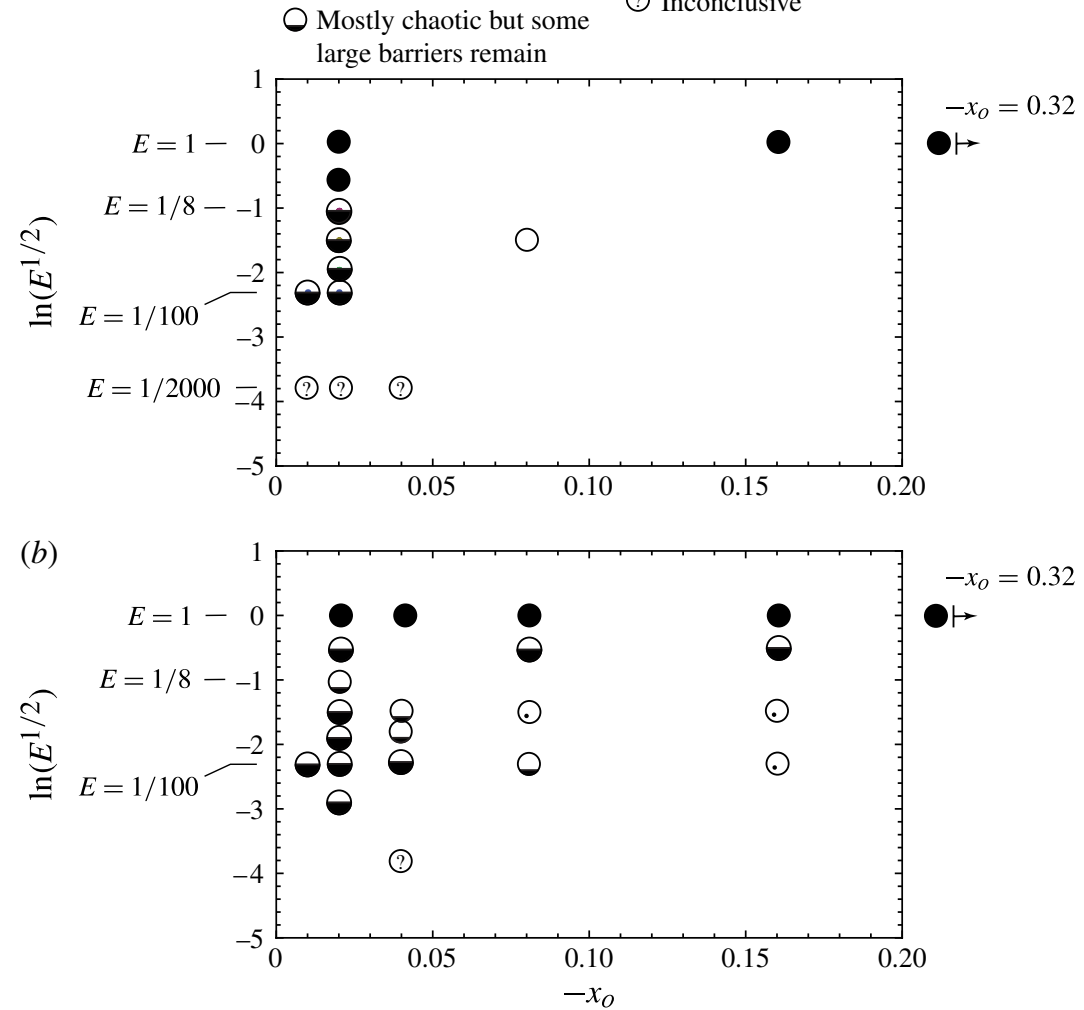

FIGURE 18. In the space of Ekman number and forcing amplitude, the symbols denote the extent to which the flow is chaotic based on visual inspection of the Poincare section (see the legend): (a) $R_{o}=0.2$ and $(b) R_{o}=1.0$. 'Inclusive' indicates that the section is filled with islands so small and barriers so thin that it is difficult to distinguish between regular and chaotic regions.

fix $E, R_{o}$ and $H / R$ and double $-x_{0}$ three times. For the largest value, $x_{0}=0.16$, only a small region of regular motion survives.

The magnitude of $x_{0}$ necessary to destroy all barriers depends on $E, R_{o}$ and $H / R$ and is difficult to define with precision since many states that are well mixed have tiny invariant islands (e.g. figure $17 d$ ). In principle, one can analyse sections showing FTLE values (e.g. figure $9 b$ ) by identifying contours with relatively low values. Our attempts yield noisy results in cases where there are many thin resonant layers, separated by thin tori, as is generally true for $E=O(1)$ and $E \ll 1$. Instead we present qualitative regime figures based on visual inspections of each Poincaré section. This survey is presented in figure 18 , with the $R_{o}=0.2$ and $R_{o}=1$ experiments in separate panels. The symbols denote qualitative behaviour ranging from no chaos (filled circles) to nearly complete chaos (open circles), as described in the legend. The most striking feature is the very strong barrier stability of the most viscous flows, particularly $E=1$. For this value we increased $-x_{0}$ to 0.32 , for both $R_{o}=0.2$ and $R_{o}=1$, without 
seeing any appreciable destruction of barriers. The $x_{0}$ threshold is much reduced once $E$ is decreased to values of $1 / 4$ or lower. Within this range most barriers are destroyed below $-x_{o}=0.08$, which roughly corresponds to the value at which the stirring rate saturates (figure $16 a$ ). For $E \ll 1$, the Poincaré sections are typically filled with islands so small and barriers so thin that it is difficult to distinguish between regular and chaotic regions. Visual inspection is therefore inconclusive, but the very small stirring rates found for these cases suggests the strong constraining presence of Taylor-Proudman dynamics.

It is clear that a direct connection between the appearance of a Poincare section and quantification of the bulk stirring is not always possible. In fact, the former can in some cases be quite deceptive.

\subsection{Stable and unstable manifolds}

To this point the discussion of stirring has focused largely on resonance width, but the chaos in the central and outer regions of the cylinder is more difficult to relate to the resonances and has, instead, been attributed to the stable and unstable manifolds emanating from the horizontally convergent and divergent points at the bottom and top of the cylinder. Since these curves are material they coincide with trajectories of the steady flow. For $E=O(1)$ or $E \ll 1$, overturning is very slow and the manifolds cycle horizontally many times over the course of a single overturning cycle. Fluid elements straddling the stable manifold are brought only occasionally into the proximity of the parabolic stagnation point at the top lid, where they tend to separate most rapidly. Stirring in the regions covered by the manifolds is therefore expected to be weakest in these cases, although our quantification of stirring rate applies to the whole volume and not just the axial and outer portions. For moderately small $E$ the manifolds have comparable azimuthal and overturning periods and fluid is brought more frequently into the hyperbolic region. (This is also the case in the dye visualization of the unstable manifold in figure $16 a$ of Fountain et al. (2000).)

\subsection{Stability of the central orbit}

The stability of the $n=1$ orbit that lies at the centre $(I=0)$ of the undisturbed tori is important. It is periodic, and therefore potentially resonant, yet the tori that surround it are typically the last to be destroyed as the disturbance amplitude increases. The case shown in figure $9(f)$ is an exception, one in which the geometry surrounding this orbit has been altered. The homoclinic ('figure-eight') geometry (figure 19a) contains two stable centres corresponding to a periodic orbit with $n=2$ (figure 19b). There is also a saddle corresponding to an orbit with $n=1$. If the value of $E$ or $R_{o}$ is changed slightly, the centres coalesce, so the $n=2$ orbit is associated with a period doubling bifurcation in either of those parameters. As the disturbance amplitude $\left|x_{o}\right|$ is diminished from 0.02 the figure-eight structure shrinks rapidly (figure $19 c, d$ ) but model resolution makes it difficult to track behaviour down to $x_{o}=0$.

The breakup of the central orbit is not governed by previous resonance analysis since the action-angle-angle coordinates are singular there. However, the overall behaviour bears some similarity to a bifurcation documented by Dullin \& Meiss (2013, figure 20) in a three-dimensional generalization of the Hénon map. There it is also confined to a narrow parameter range.

\subsection{Aspect ratio}

Most oceanographic applications have $H / R$ much less than the $O(1)$ values used above. We have performed a limited number of simulations with $H / R$ values down to $1 / 50$, 
(a)

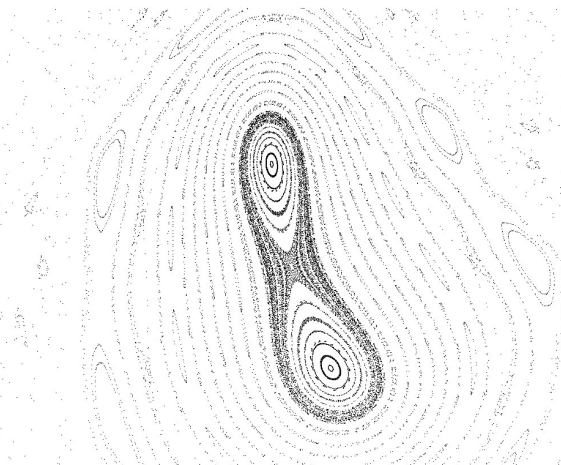

(c)

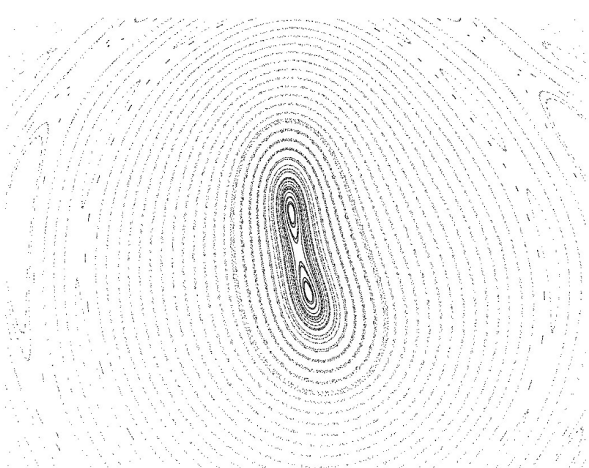

(b)

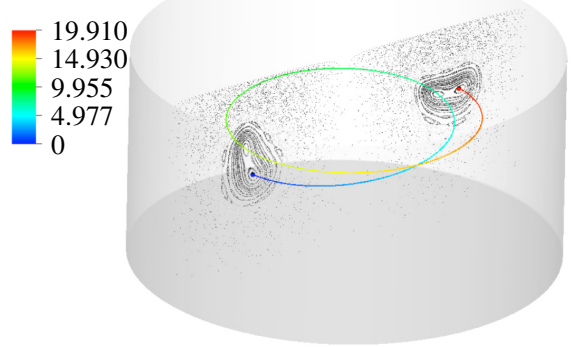

$(d)$

FIGURE 19. (Colour online) Blown-up portion of figure $10(f)$ showing the homoclinic pattern resulting form the period doubling bifurcation of the central periodic orbit of the undisturbed flow. Also shown, at the same scale, are sections that result when the amplitude of the forcing is reduced. In the final frame the periodic $(n=2)$ trajectory corresponding to the centres of the 'figure-eight' geometry: $(a, b) x_{o}=-0.02 ;(c) x_{o}=-0.01 ;(d) x_{o}=-0.009$.

realistic for shallow, submesoscale or small mesoscale eddies. The results do not differ qualitatively with those already shown, and the Lagrangian regimes for weak forcing obtained can be predicted largely on the basis of the anticipated typical values of $T_{\theta} / T_{\phi}$. This, in turn, depends on what is held fixed while $H / R$ is changed. Since the dimensionless cylinder radius is fixed at unity, a decrease in $H / R$ is accomplished through a decrease in the dimensionless depth. If $E$ and $R_{o}$ are held steady, this process leaves the thickness-to-depth ratio of the Ekman layers fixed but decreases the vertical excursion time of fluid parcels. Thus, $T_{\phi}$ decreases while $T_{\theta}$ remains the same, and the minimum possible $n$ in (3.7) decreases. On the other hand, fixed values of $R_{o}$ and $R_{e}$ imply that $E$ increases as $H / R$ decreases. In figure 20 the values of $R_{o}$ and $R_{e}$ remain fixed while $H / R$ is changed from unity to $1 / 50$. According to (2.4) this raises $E$ from $1 / 16384$ to 0.15 , implying a transition from relatively thin Ekman layers to moderately thick ones. The regime does in fact switch from type III (rigid interior with thin resonant layers) to type I (rounded tori, also with thin resonant layers), as expected from the above arguments.

We note one other result of a decrease in aspect ratio. As shown in the lower frame of figure 20, a homoclinic structure has appeared near the centre of the nested tori. Although it resembles the feature described in $5 e$, it is much larger. Such structures 
(a)

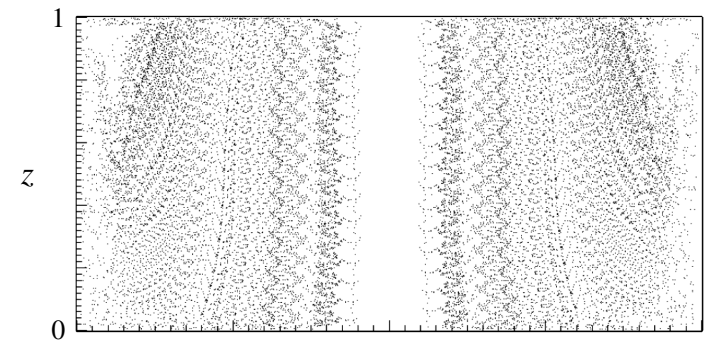

(b)

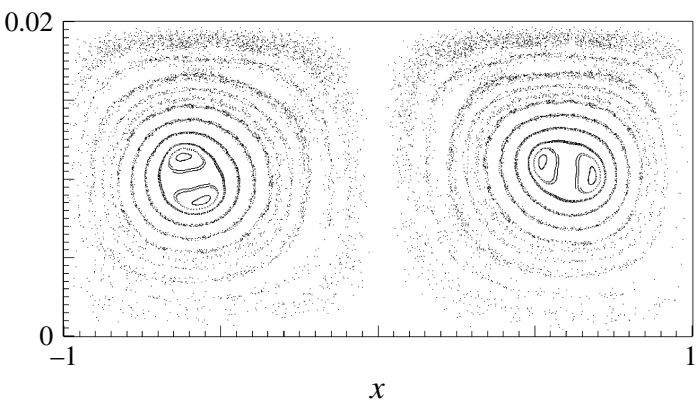

FIGURE 20. An example of the results of decreasing the horizontal aspect ratio $H / R$ from 1 (a) to $1 / 50(b)$ for the case $R_{o}=0.5$ and $R_{e}=8192$. The corresponding $E$ are $1 / 16384$ and 0.15 . The bottom frame has been vertically stretched for visual purposes. Note that there is very weak time dependence in both flows.

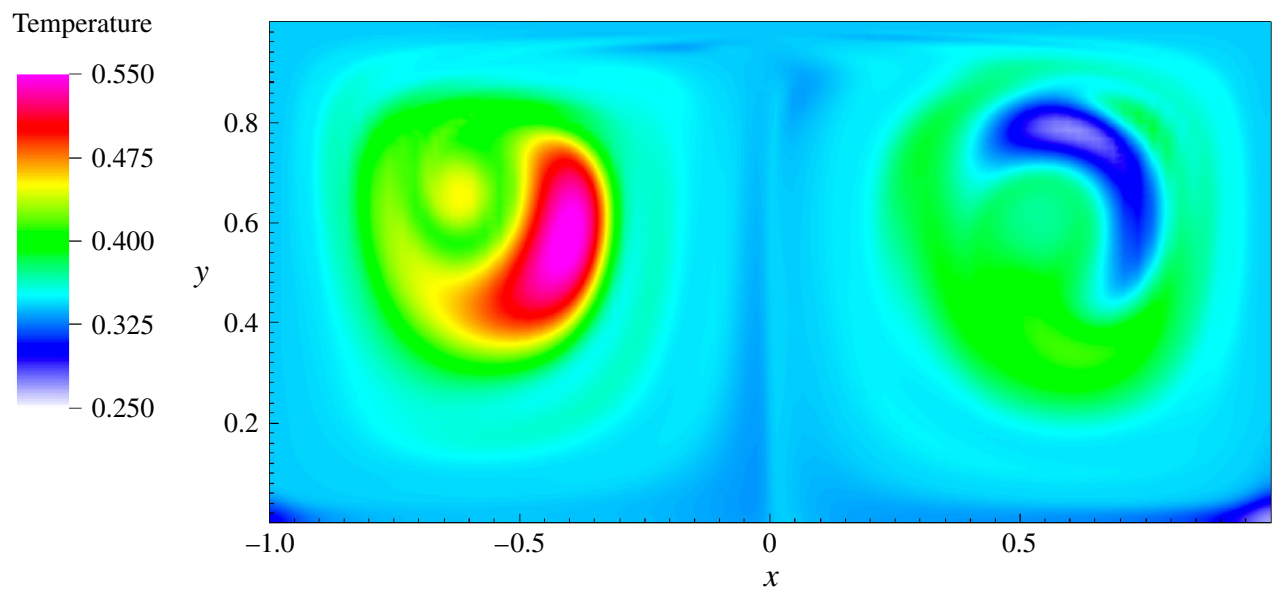

FIgURE 21. (Colour online) The tracer distribution corresponding to the flow used to produce the Poincare section shown in figure 10(i) and plotted over the same section. The plot has been made at $t=300$, which is long after diffusion has begun to homogenize the field. The tracer evolution also corresponds to that shown in figure 15, but plotted over a different section.

appear to be more common at small values of $H / R$, though they are rarely large. We have not explored this aspect further. 


\section{Applications to the ocean}

Non-uniform mixing, transport barriers, islands, etc. would strongly influence distributions of oceanographically relevant physical, chemical and biological tracers. However, a number of complications must be addressed before direct comparisons with ocean features can be meaningful. These include the effects of stratification, time-dependence, interactions with neighbouring eddies and the diffusive effects of background turbulence. Each subject merits a substantial investigation. The goal of the present work has been to provide a benchmark that can be used as a jumping-off point for such studies. Observational challenges are also considerable due to sparse sampling and to the difficulty of measuring vertical velocities in the ocean.

We can anticipate some of the issues that will arise in dealing with these realworld issues. On the subject of time dependence, Cheng \& Sun (1990) show that in volume-preserving systems such as ours, some invariant tori will survive a timeperiodic perturbation of small amplitude. Preliminary studies using our kinematic and Navier-Stokes models have confirmed the existence of time-periodic tori. For more complex time dependence, investigations are largely restricted to two dimensions. Barriers are also found here, but without the fine scale structures such as islands (e.g. Haller \& Poje 1998; Rogerson et al. 1999; Miller et al. 2002; Rypina et al. 2011; Haller \& Beron-Vera 2012). It is quite possible that such will be the case with time-aperiodic flows in three dimensions.

Of equal importance are the effects of the unresolved turbulence, inevitable in any ocean model. Since full resolution of turbulence for ocean eddies is beyond the capability of current models, one must rely on turbulent parameterizations to estimate the diffusive effects on tori and other barriers. Approaches range from adding noise to trajectories to large eddy simulations and are beyond the scope of the current study. However, the tracer diffusivity specified in our tracer release experiments introduces one element of turbulence, and it is worth considering the extent to which tori or islands show up in the tracer fields after the diffusive stage of evolution is underway. A case in point appears in figure 21, which shows the tracer field at $t=300$ for the flow used to produce the Poincaré section of figure 10(i). The latter contains a single prominent island with $n=1$, along with a nest of tori that surrounds the original central orbit. Both of these features, and especially the large island, are captured in the figure 21 tracer field. So, despite the fact that much of the tracer variance has been eroded by diffusion, the barriers still have a presence. The island structure can be seen with more clarity in a video of the tracer evolution, available at https://www. dropbox.com/sh/i44jwhykr1loj70/0e8FmqR_aR?n=20522545. Although the images lack the clarity and resolution of Poincaré sections or pinpoint dye release, they apparently have the potential to reveal the most prominent Lagrangian structures. This result also provides hope that some barriers may be detectable in the field using tracer release experiments (Sundermeyer et al. 2007).

These challenges notwithstanding, one can attempt to identify eddy scales over which the stirring mechanism discussed herein could plausibly apply. Since the manifestations of chaos are felt over many Lagrangian cycles of motion, the eddy in question must live sufficiently long for fluid parcels to overturn many times. This would appear to eliminate large, mesoscale eddies (e.g. Gulf Stream rings) from consideration. With typical upwelling/downwelling rates or $10-40 \mathrm{~cm} \mathrm{day}^{-1}$, and with depths of $500-1000 \mathrm{~m}$, the overturning times are typically measured in months or years, the same scale as the life of the feature. Ledwell et al. (2008) infer upwelling rates of around $0.4 \mathrm{~m} \mathrm{day}^{-1}$ in a $700 \mathrm{~m}$ deep mode water eddy, but again the overturning times are several years. In addition, these eddies have tiny Ekman numbers 
and our results indicate that chaotic stirring will be weak relative to shallower features. A similar limitation holds for hurricanes, which have rapid updrafts but very slow rates of subsidence. The overturning time of an air parcel is of the order of the life time of the storm.

For the ocean surface submesoscale, which roughly includes scales ranging from hundreds of meters up to the Rossby radius of deformation, typically $8-30 \mathrm{~km}$, the vertical velocities can reach $2 \mathrm{~cm} \mathrm{~s}^{-1}$, so with depths on the order of $20-50 \mathrm{~m}$, the overturning times can range from hours to days. Thus, submesoscale features that are able to remain coherent for several days would qualify. Ekman numbers for these cases would lie in the range 0.01-1 and our results indicate that chaotic stirring is larger for these cases. Perhaps the most interesting among these findings are cases where $E=O(1)$ and the disturbance amplitude is large, which is perhaps most realistic for the ocean. Stirring rates for this case are the largest of all considered, though questions remain as to the relative importance of chaos and shear.

Ocean eddies are often generated by a barotropic or baroclinic instability mechanism acting within a current. Once formed, the eddies maintain their vorticity over scales ranging from days to years. The horizontal vorticity is set during the formation stage, and not driven by a surface stress as in our model. However, wind stress does generate an overturning circulation within them. Ledwell et al. (2008) point out that a uniform wind blowing over an eddy will create a surface stress of largest magnitude where the current opposes the wind. The resulting stress divergence will lead to an upwelling/downwelling pattern that has the structure of a low azimuthal mode. The disturbances imposed in our model are also contained in a low azimuthal mode, although the spatial pattern differs. So, although the forcing mechanisms differ, the overall Eulerian features of the observed and modelled flows bear similarity.

Other intriguing applications include the deep convection cells that occur in winter in the Labrador Sea, the northern Mediterranean, and the Nordic Seas. Marshall \& Schott (1999) gave review of model results and observations. With their weak stratification, $O(1)$ aspect ratios, Rossby numbers in the range 0.1-0.2, vertical velocities 5-10 $\mathrm{cm} \mathrm{s}^{-1}$ and depth scales of $1-2 \mathrm{~km}$, these features have much in common with the circulations modelled here, and overturning can occur several times in the course a day. They are also unstable and may break up and/or interact with neighbouring cells, but chaotic advection may be relevant during the formation stage. These cells are forced by a buoyancy loss to the atmosphere, and not by wind stress, so any model must replace the top Ekman layer of our cylinder flow with a rotational buoyancy layer. However, the net vertical volume transport in models of convection cells is very small compared with the overturning transport, so the overall overturning circulations of the two cases may be similar.

\section{Discussion}

Using a combination of analytical and numerical techniques, we have constructed a scenario that explains some, but certainly not all, aspects of chaotic stirring in a threedimensional cylinder flow in which Ekman dynamics are crucial. The investigation is carried out over a parameter range that is oceanographically relevant for eddies ranging from submesoscale to mesoscale. This is one aspect that distinguishes the Eulerian dynamics considered in our studies from earlier work. In particular, moderate to strong background rotation plays a dominant role in our study.

For weak disturbance amplitude, the observed extent and measured intensity of chaotic stirring is consistent with expectations based on analytical Ekman layer 
solutions. For $E=O(1)$ viscous Ekman layers extend through the water column, the top and bottom are in viscous contact, the vertical velocity is suppressed, and the overturning time period is long. The azimuthal velocity is $O(1)$ for all $E$ and therefore trajectories cycle horizontally many times over each overturning cycle. Resonances produced under these conditions contain braided chains with many small islands. The structures of resulting high-wavenumber, periodic trajectories do not project efficiently onto the forced disturbance, which varies gradually in space. The result is that the chaos generated around resonant tori is confined to very thin layers, as suggested by (3.13).

When the cylinder depth is increased moderately (or the viscosity reduced), the top and bottom Ekman layers separate. The top and bottom are no longer in strong viscous contact and there is more room for stronger vertical motion to develop and the typical overturning time decreases markedly. Some periodic trajectories execute only 1-3 azimuthal cycles over each period and this simplified structure leads to a stronger resonance with disturbance. Resonant layers are thicker and the stirring rate (for weak forcing) is greater. As the cylinder is made even deeper the dimensional vertical velocity approaches a fixed value and so the overturning time lengthens, the interior become more vertically rigid, resonant layers become thinner, and the stirring rate decreases.

For the chaotic region generated by the breakup of the vertical streamline at $r=0$, the qualitative structure of the stable and unstable manifolds over one or two overturning cycles mirror the trajectory structures described above. For $E=O(1)$ or $E \ll 1$, fluid parcels lying close to the unstable manifold will cycle rapidly in the horizontal direction but will be brought into the proximity of the top lid 'stagnation' point only occasionally. Nearby trajectories can be expected to undergo slower separation in these cases than for $E$ in the range $1 / 20<E<1 / 100$, where overturning is more rapid and parcels encounter the divergent stagnation region more frequently.

The observed peaks in the bulk stirring rate for various cases of weak forcing are proximal to the peak in the vertical velocity predicted by a linear Ekman layer theory.

Interestingly, some of the above trends disappear when the forcing amplitude becomes large. Most significantly, cases with $E=O(1)$, which have very small stirring rates at small amplitude, experience rapid increase in stirring, despite the fact that chaos remains confined to thin resonant layers. At moderately small $E$, the stirring rates saturate as the forcing amplitude increases. Saturation roughly coincides with the destruction of the most prominent barriers and the onset of nearly global chaos. For very small $E$, the increase in disturbance amplitude has little effect on the stirring rates, which remain the lowest of all settings. The overall trend for large disturbances, then, is that the stirring rate decreases monotonically from a maximum at $E=O(1)$ down to $E=1 / 2000$.

This work also identifies several challenges for theory. The behaviour of the stable and unstable manifolds resulting from breakup of streamline at $r=0$ intuitively suggests chaos, but we have been unable to find a body of work that formalizes this result or that suggest any value in computing the manifolds. Similarly, the horizontal orbit that lies at the centre of the nested tori in the undisturbed state is periodic and therefore potentially resonant. However our studies suggest stability, even at large disturbance amplitude, for all but a small region in $\left(E, R_{o}\right)$ parameter space. Further inquiry is hindered by the fact that the action-angle-angle variable set is singular there. Dullin \& Meiss (2013) find similar behaviour for a different system. Finally, one must ask what sort of analysis toolset is available for three-dimensional flows with complex 
time dependence, where Poincaré sections are not relevant. FTLEs currently provide the most popular approach in two-dimensional, but detailed agreement with Poincaré sections in our steady, three-dimensional flow requires integration times equivalent to hundreds or differential rotation periods. Thus, the interpretation of FTLEs could be tricky in three-dimensional flows undergo even moderately rapid time evolution.

The results from this study certainly create the possibility that hidden, complex Lagrangian structures may exists in oceanic eddies. The corresponding stirring and mixing processes could have significant implications for how heat and salt and other quantities of critical importance for the climate dynamics are transported in threedimensional within the global-scale ocean circulation.

\section{Acknowledgements}

L.J.P., I.I.R., T.M.Ö. and P.W. have been supported on DOD (MURI) grant N000141110087, administered by the Office of Naval Research. I.I.R. and L.J.P. received additional support from Grant NSF-OCE-0725796 from the National Science Foundation.

\section{Appendix A. Linear solutions for undisturbed states}

\section{A.1. Scaling}

Let $R$ and $H$ denote the dimensional cylinder radius and depth, and let $\delta \Omega$ be a scale for the differential lid rotation. Then $U=\delta \Omega R$ and $P=\rho H \Omega U$ can serve as velocity and pressure scales, suggesting the following non-dimensionalizations:

$$
\boldsymbol{u}^{*}=U \boldsymbol{u}=R_{o} \Omega \boldsymbol{u}, \quad(x, y, z)^{*}=(x R, y R, z H), \quad t^{*}=\Omega t, \quad p^{*}=P p
$$

where asterisks denote dimensional quantities and $R_{o}=\delta \Omega / \Omega$. For linear $\left(R_{o} \ll 1\right)$, steady, azimuthally uniform $(\partial / \partial \theta=0)$ flow with $H=R$, the governing equations (Greenspan 1969) in cylindrical coordinates are

$$
\begin{gathered}
-2 v=-\frac{\partial p}{\partial r}+E\left(\nabla^{2}-\frac{1}{r^{2}}\right) u \\
2 u=E\left(\nabla^{2}-\frac{1}{r^{2}}\right) v \\
0=-\frac{\partial p}{\partial z}+E \nabla^{2} w \\
\frac{\partial u}{\partial r}+\frac{u}{r}+\frac{\partial w}{\partial z}=0 .
\end{gathered}
$$

\section{A.2. Asymptotic solution with zero lid velocity/stress at outer rim}

The boundary conditions require that the zonal, azimuthal and vertical velocity components $(u, v, z)$ vanish at the cylinder wall $(r=1)$ and at the bottom $z=0$. At the top lid $(z=1), u=w=0$ but $v=v_{T}(r)$, where $v_{T}(r) \rightarrow 0$ as $r \rightarrow 1$. Since $v$ goes to zero at the outer edge of the lid, there is no radial Ekman flux there and Ekman layer must return fluid downward into the interior before it reaches the outer wall. There is no need for sidewall (Stewardson) layers to close the overturning circulation or bring the azimuthal velocity to zero. Strictly speaking, a sidewall layer is required to bring $w$ to zero there, but if this requirement is ignored the asymptotic solution for $E \ll 1$ is

$$
u(r, z)=\frac{v_{T}(r)}{2}\left[\sin \xi_{T} \mathrm{e}^{-\xi_{T}}-\sin \xi_{B} \mathrm{e}^{-\xi_{B}}\right]+\frac{E}{4 r} \frac{\partial}{\partial r}\left(r \frac{\partial v_{T}}{\partial r}\right)
$$


Chaotic advection in a steady, three-dimensional, Ekman-driven eddy

$$
\begin{gathered}
v(r, z)=\frac{v_{T}(r)}{2}\left[1+\cos \xi_{T} \mathrm{e}^{-\xi_{T}}-\cos \xi_{B} \mathrm{e}^{-\xi_{B}}\right] \\
w(r, z)=\frac{E^{1 / 2}}{4 r} \frac{\partial}{\partial r}\left(r v_{T}(r)\right)\left[1-\left(\sin \xi_{T}+\cos \xi_{T}\right) \mathrm{e}^{-\xi_{T}}-\left(\sin \xi_{B}+\cos \xi_{B}\right) \mathrm{e}^{-\xi_{B}}\right] .
\end{gathered}
$$

Note that for the boundary condition (2.4), $v_{T}(r)=4 r(1-r)$.

\section{A.3. Flow between to infinite plates, arbitrary $E$}

If we return to the traditional case with $v_{T}=r$ and consider a domain that is unbounded in $r$, then it is not difficult to solve the problem for arbitrary $E$. We will also seek a solution that is many Ekman thicknesses away from the central axis $\left(r * \gg(v / \Omega)^{1 / 2}\right)$ so that $\partial / \partial r \ll \partial / \partial z$ and $1 / r \ll \partial / \partial z$. Then (A 2)-(A 4) become

$$
-2 v=-\frac{\partial p}{\partial r}+E \frac{\partial^{2} u}{\partial z^{2}}, \quad 2 u=E \frac{\partial^{2} v}{\partial z^{2}} \quad \text { and } \quad 0=-\frac{\partial p}{\partial z}+E \frac{\partial^{2} w}{\partial z^{2}} . \quad(\text { A } 9 a-c)
$$

Elimination of $p$ and $v$ from this set and from (A 5) leads to

$$
E^{2}\left(\frac{\partial^{4} u}{\partial z^{4}}+\frac{\partial}{\partial r}\left(\frac{1}{r} \frac{\partial}{\partial r}\left(r \frac{\partial^{2} u}{\partial z^{2}}\right)\right)\right)+4 u=0 .
$$

The top lid boundary condition suggests solutions proportional to $r$ so we take $v=r V(z)$, and by extension $u=r U(z)$ (where $U$ is not to be confused with the previous velocity scale). Equation (A 10) then reduces to

$$
\frac{\partial^{4} U}{\partial z^{4}}+4 E^{-2} U=0
$$

with $U(0)=U(1)=0$. The boundary conditions and requirement of zero net radial volume flux suggest a solution that is antisymmetric about the mid-depth $(z=1 / 2)$ :

$$
U(z)=A \sin (\zeta) \cosh (\zeta)+B \cos (\zeta) \sinh (\zeta)
$$

where $\zeta=(z-1 / 2) / E^{1 / 2}$.

Integration of (A $9 b)$ then provides a solution for $V$, which is

$$
V(z)=C_{1}+B \sin (\zeta) \cosh (\zeta)-A \cos (\zeta) \sinh (\zeta)
$$

Finally, the vertical velocity can be found from integration of (A 9c):

$$
w(r, z)=-E^{1 / 2}\left\{\begin{array}{c}
A[\sin (\zeta) \sinh (\zeta)-\cos (\zeta) \cosh (\zeta)] \\
+B[\cos (\zeta) \cosh (\zeta)+\sin (\zeta) \sinh (\zeta)]-D
\end{array}\right\}
$$

Satisfaction of the boundary conditions $w(0)=w(1)=0, v(0)=0, v(1)=r$, and $u(1)=u(0)=0$ leads to

$$
\begin{gathered}
A=-\frac{1}{2} \frac{c S}{s^{2} C^{2}+c^{2} S^{2}}, \quad B=\frac{1}{2} \frac{s C}{s^{2} C^{2}+c^{2} S^{2}}, \quad C_{1}=\frac{1}{2}, \\
D=A(s S-c C)+B(c C+s S) \\
\left.\begin{array}{c}
S=\sin \left(\frac{1}{2 E^{1 / 2}}\right), \quad c=\cos \left(\frac{1}{2 E^{1 / 2}}\right), \\
S=\sinh \left(\frac{1}{2 E^{1 / 2}}\right), \quad C=\cosh \left(\frac{1}{2 E^{1 / 2}}\right) .
\end{array}\right\}
\end{gathered}
$$




\section{Appendix B. Kinematic model}

It is convenient to use both Cartesian coordinates $(x, y, z)$, with corresponding velocity components $\left(v^{(x)}, v^{(y)}, w\right)$, and cylindrical coordinates $(r, \theta, z)$ with velocity components $(u, v, w)$. The former are required in order to specify a finite and welldefined velocity at the origin $r=0$. The kinematic model is given by

$$
\begin{aligned}
v^{(x)} & =V^{(x)}(x, y, z)+\tilde{v}^{(x)}(x, y, z, t), \\
v^{(y)} & =V^{(y)}(x, y, z)+\tilde{v}^{(y)}(x, y, z, t), \\
w & =W(x, y, z)+\tilde{w}(x, y, z, t) .
\end{aligned}
$$

The azimuthally symmetric background state is defined

$$
\begin{aligned}
V^{(x)}(x, y, z) & =-\frac{x}{3}(1-2 z)(a-r)-\alpha y, \\
V^{(y)}(x, y, z) & =-\frac{y}{3}(1-2 z)(a-r)+\alpha x, \\
W(x, y, z) & =z(1-z)\left(\frac{2}{3} a-r\right),
\end{aligned}
$$

and the cylinder boundaries lie at $z=0, z=1$ and $r=a$, where $a$ is the nondimensional radius. This set is equivalent to (2.8).

The perturbation to the background is contained entirely in the horizontal velocity and is described by the streamfunction $\tilde{\psi}$ given by (2.10). The velocity perturbations are therefore

$$
\begin{aligned}
\tilde{v}^{(x)}= & -\frac{\partial \tilde{\psi}}{\partial y}=\varepsilon_{1}(1-\beta z) y\left(x-x_{o}\right) \\
& -\frac{1}{2} \varepsilon_{2}(1-\beta z)\left[2 y\left(a^{2}-2 r^{2}\right) \cos \left(n_{\theta} \theta\right)-n_{\theta} x\left(a^{2}-r^{2}\right) \sin \left(n_{\theta} \theta\right)\right] \\
\tilde{v}^{(y)}= & \frac{\partial \tilde{\psi}}{\partial x}=\varepsilon_{1}(1-\beta z)\left[\frac{1}{2}\left(a^{2}-r^{2}\right)-x\left(y-x_{o}\right)\right] \\
& +\frac{1}{2} \varepsilon_{2}(1-\beta z)\left[2 x\left(a^{2}-2 r^{2}\right) \cos \left(n_{\theta} \theta\right)+n_{\theta} y\left(a^{2}-r^{2}\right) \sin \left(n_{\theta} \theta\right)\right]
\end{aligned}
$$

and $\tilde{w}=0$.

\section{Appendix C. Action-angle-angle coordinates}

Following Mezic \& Wiggins (1994, (31)-(34)) we equate $I$ with the cross-sectional area in the $(r, z)$ plane of the torus in question. For each $I$, we define an anglelike coordinate $\phi$ that measures meridional position, as suggested in figure 4. The coordinate $\phi$ is defined as

$$
\phi=\frac{2 \pi}{T_{\phi}(I)} t \quad\left(0 \leqslant t \leqslant T_{\phi}(I)\right),
$$

where $T_{\phi}=2 \pi / \Omega_{\phi}(I)$ is the period, and $\Omega_{\phi}(I)$ the frequency, of motion for an orbit around the torus (in the $r-z$ plane). To establish $\phi$ one must first calculate the period $T_{\phi}$, by following a trajectory (beginning at some reference values of $z$ and $r$ ) through one cycle in the $(r, z)$ plane. Then $\phi$ is defined to vary linearly with time from zero to $2 \pi$ over this circuit. Note that $\phi$ is generally not a meridional angle, measured (say) 
from the centre of the tori. It is rather a stretched, angle-like coordinate measuring position around the tube of the torus and remains well defined even when the tube surface is complicated. (It is usually difficult to relate $\phi$ to $r$ and $z$ analytically; to do so, one must to solve for the positions $r(t)$ and $z(t)$ as the trajectory winds round the torus, invert these relate these relations to get $t(r, z)$, then substitute into (C1).) The equations governing trajectories in the undisturbed state can now be written as

$$
\frac{\mathrm{d} \theta}{\mathrm{d} t}=\frac{v(I, \phi)}{r(I, \phi)}, \quad \frac{\mathrm{d} \phi}{\mathrm{d} t}=\Omega_{\phi}(I) \quad \text { and } \quad \frac{\mathrm{d} I}{\mathrm{~d} t}=0 .
$$

The definition of a period of motion in the $\theta$-direction is more difficult since the period between successive crossings of the $(r, z)$ plane is not constant. However, a fixed period $T_{\theta}$ can be defined in terms of a new azimuthal coordinate:

$$
\tilde{\theta}=\theta-\theta_{o}(\phi, I),
$$

where

$$
\theta_{o}(\phi, I)=\int_{\phi} \frac{v\left(I, \phi^{\prime}\right)}{r\left(I, \phi^{\prime}\right) \Omega_{\phi}(I)} \mathrm{d} \phi^{\prime}-\frac{\phi}{2 \pi} \int_{0}^{2 \pi} \frac{v(I, \phi)}{r(I, \phi) \Omega_{\phi}(I)} \mathrm{d} \phi .
$$

Thus, $\tilde{\theta}$ is just a shifted version of $\theta$, with the origin depending on $\phi$ for a particular torus $I$ (see figure 4 ). It can easily be verified that (C $2 a$ ) now becomes

$$
\frac{\mathrm{d} \tilde{\theta}}{\mathrm{d} t}=\int_{0}^{2 \pi} \frac{v(I, \phi)}{r(I, \phi)} \mathrm{d} \phi=\Omega_{\theta}(I),
$$

so that a period $T_{\theta}(I)=2 \pi / \Omega_{\theta}(I)$, equal to the time between successive zero crossings of $\tilde{\theta}$, can be defined for all trajectories on a torus. In the new (non-orthogonal) coordinate set $(\phi, \tilde{\theta}, I)$, the equations for trajectories in the undisturbed flow are given by $(\mathrm{C} 2 b, c)$ and (C 5).

\section{Appendix D. Verification of tendencies in the resonance width formula (3.13)}

In order to check the dependence of $\Delta I$ on disturbance amplitude, we have carried out a sequence of experiments based on (2.8) and (2.10), and for which the parameter $\varepsilon_{1}$, which functions as the amplitude parameter $\varepsilon$ above, is varied. As $\varepsilon_{1}$ increases, the width of the four-island chain (figure 5) increases and the corresponding $\Delta I$ is calculated as the difference in cross-sectional area between the undisturbed tori whose positions correspond to the outer extrema of the islands. As shown in figure $22(a)$ the results show behaviour very close to the expected $\sqrt{\varepsilon_{1}}$ dependence.

Isolation of the dependence of $\Delta I$ on $\mathrm{d} \Omega_{\phi} / \mathrm{d} I$ or $\mathrm{d} \Omega_{\theta} / \mathrm{d} I$ is more difficult owing to the difficulty in fixing $n$ and $I$ as these frequency gradients are varied. In the kinematic model, the azimuthal velocity varies linearly with $r, \Omega_{\theta}$ is constant, and therefore $\mathrm{d} \Omega_{\theta} / \mathrm{d} I=0$, so we only explore the dependence on $\mathrm{d} \Omega_{\phi} / \mathrm{d} I$. The first equality in (3.13) suggest that $\Delta I$ varies in proportion to $\left(\mathrm{d} \Omega_{\phi} / \mathrm{d} I\right)^{-1 / 2}$. To facilitate this calculation we generalize the unperturbed state (2.6) by adding an amplitude factor $A$ to the overturning circulation. The undisturbed velocity field, now expressed in cylindrical coordinates, becomes

$$
\begin{aligned}
u & =-A \frac{(1-2 z) r(R-r)}{3} \\
v & =\alpha \\
w & =-A z(1-z)\left(\frac{2}{3} r-R\right) .
\end{aligned}
$$



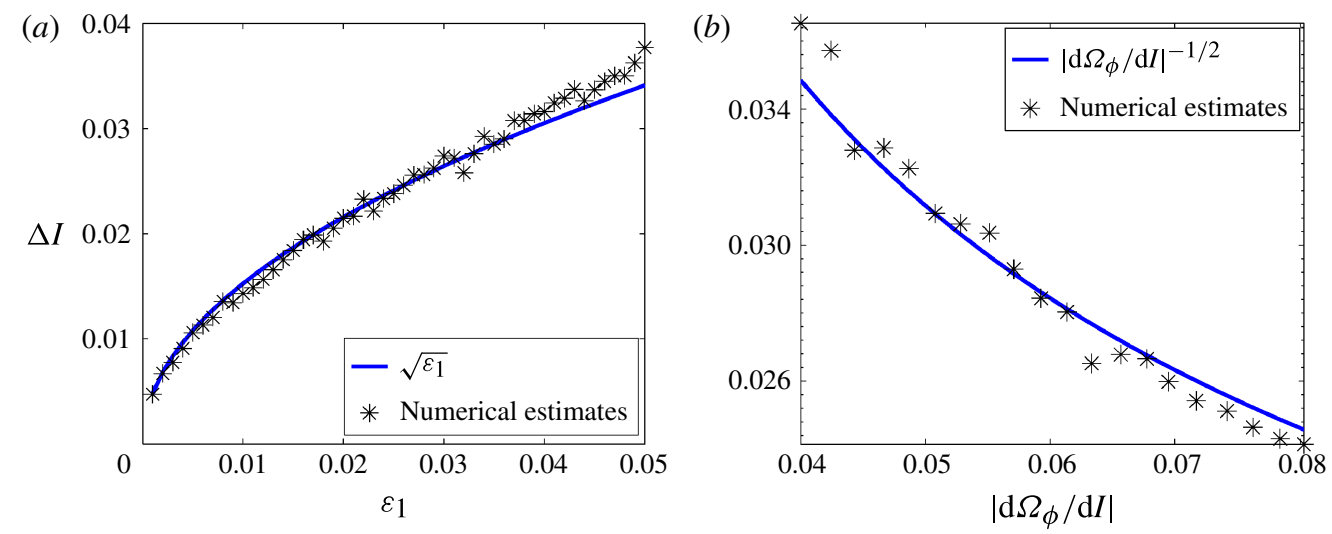

FIgURE 22. (Colour online) Test of tendencies contained in the resonant width formula (3.13). (a) Plot of resonance width $\Delta I$ versus the square root of forcing amplitude $\varepsilon_{1}$ for the period 4 resonant layer/island chain seen in the kinematic model with parameters as in figure 5: $\alpha=0.35, y_{o}=-0.25, \beta=1$ and $\varepsilon_{2}=\varepsilon_{3}=0$. (b) Plot of $\Delta I$ versus $\left|\mathrm{d} \Omega_{\phi} / \mathrm{d} I\right|^{-1 / 2}$ based on the kinematic model with the modified unperturbed state given by (D 1)-(D 3). The model parameters are the same as in $(a)$ but with $\varepsilon_{1}=0.025$ and variable $\alpha$.

For our standard choice of parameters $A=1$ and $\alpha=0.35$, a resonant torus with $n=4$ is present. We now change both parameters through 20 different pairings in a way that preserves the $I$ value of the $n=4$ torus but allows $\mathrm{d} \Omega_{\phi} / \mathrm{d} I$ to vary. As shown in figure $22(b)$ the resulting $\Delta I$ are consistent with a $\left|\mathrm{d} \Omega_{\phi} / \mathrm{d} I\right|^{-1 / 2}$ dependence.

\section{REFERENCES}

AREF, H. 1984 Stirring by chaotic advection. J. Fluid Mech. 143, 1-21.

BArcilon, V. \& Pedlosky, J. 1967 A unified theory of homogeneous and stratified rotating fluids. J. Fluid Mech. 29, 609-621.

Behringer, R. P., Meyers, S. D. \& Swinney, H. L. 1991 Chaos and mixing in a geostrophic flow. Phys. Fluids A 3, 1234-1249.

Beron-Vera, F. J. \& Olascoaga, M. J. 2009 An assessment of the importance of chaotic stirring and turbulent mixing in the West Florida Shelf. J. Phys. Oceanogr. 9, 1743-1755.

BRANicki, M. \& KirWAN, A. D. 2010 Stirring: the Eckart paradigm revisited. Intl J. Engng Sci. 48, $1027-1042$.

Cheng, C.-Q. \& SUN, Y.-S. 1990 Existence of invarient tori in three-dimensional measurepreserving mappings. Celestial Mech. 47, 275-292.

Chirikov, B. V. 1979 A universal instability of many-dimensional oscillator systems. Phys. Rep. 52, 265-379.

Coulliette, C., Lekien, F., Paduan, J. D., Haller, G. \& Marsden, J. E. 2007 Optimal pollution mitigation in Monterey Bay based on coastal radar data and nonlinear dynamics. Environ. Sci. Technol. 41, 6562-6572.

Coulliette, C. \& Wiggins, W. 2001 Intergyre transport in a wind-driven, quasigeostrophic double gyre: an application of lobe dynamics. Nonlinear Process. Geophys. 8, 69-94.

D'Asaro, E. A. 2003 Performance of autonomous Lagrangian floats. J. Atmos. Ocean. Technol. 20, 896-911.

Deese, H. E., Pratt, L. J. \& Helfrich, K. R. 2002 A laboratory model of exchange and mixing between western boundary layers and subbasin recirculation gyres. J. Phys. Oceanogr. 32, $1870-1889$. 
Del-Castillo-Negrete, D. \& Morrison, P. J. 1993 Chaotic transport by Rossby waves in shear flow. Phys. Fluids A 5 (4), 948-965.

Dijkstra, H. A. \& Katsman, C. A. 1997 Temporal variability of the wind-driven quasigeostrophic double gyre ocean circulation: basic bifurcation diagrams. Geophys. Astrophys. Fluid Dyn. 85, 195-232.

Dombre, T., Frisch, U., Green, J. M., Henon, M., Mehr, A. \& Soward, A. M. 1986 Chaotic streamlines of the ABC flows. J. Fluid Mech. 353-391.

DuAn, J. Q. \& Wiggins, S. 1996 Fluid exchange across a meandering jet with quasi-periodic time variability. J. Phys. Oceanogr. 26, 1176-1188.

Dullin, H. R. \& MeIss, J. D. 2013 Quadratic volume-peserving maps: invariant circles and bifurcations. SIAM J. Appl. Dyn. Syst. 8 (1), 76-128.

FISCHER, P. F. 1997 An overlapping Schwarz method for spectral element solution of the incompressible Navier-Stokes equations. J. Comput. Phys. 133, 84-101.

Fountain, G. O., Khakhar, K. V., Mezic, I. \& Ottino, J. M. 2000 Chaotic mixing in a bounded three-dimensional flow. J. Fluid Mech. 265-301.

Greenspan, H. P. 1969 The Thoery of Rotating Fluids. Cambridge University Press.

HALler, G. \& Beron-Vera, F. J. 2012 Geodesic theory of transport barriers in two-dimensional flows. Physica D 241, 1680-1702 doi:10.1016/j.physd.2012.06.012.

Haller, G. \& Poje, A. C. 1998 Finite time transport in aperiodic flows. Physica D 119, 352-380.

HART, J. E. \& Kittelman, S. 1996 Instabilities of the sidewall boundary layer in a differentially driven rotating cylinder. Phys. Fluids 8, 692-696.

Haza, A. C., Griffa, A., Martin, P., Molcard, A., Özgökmen, T. M., Poje, A. C., Barbanti, R., Book, J. W., Poulain, P. M., Rixen, M. \& Zanasca, P. 2007 Model-based directed drifter launches in the Adriatic Sea: results from the DART experiment. Geophys. Res. Lett. 34, L10605 doi:10.1029/2007GL029634.

Haza, A., Özgökmen, T., Griffa, A., Molcard, A., Poulain, P. \& Peggion, G. 2010 Transport properties in small scale coastal flows: relative dispersion from VHF radar measurements in the Gulf of La Spezia. Ocean Dyn. 60, 861-882.

Haza, A. C., Poje, A., Özgökmen, T. M. \& Martin, P. 2008 Relative dispersion from a highresolution coastal model of the Adriatic Sea. Ocean Model. 22, 48-65.

ILICAK, M., ÖZGÖKMEN, T., Özsöy, E. \& FISCHER, P. 2009 Non-hydrostatic modelling of exchange flows across complex geometries. Ocean Model. 29, 159-175.

Joseph, B. \& Legras, B. 2002 Relation between kinematic boundaries, stirring, and barriers for the Antarctic Polar Vortex. J. Atmos. Sci. 59, 1198-1212.

Kruznetsov, L., Toner, M., Kirwan, A. D. Jr, Jones, C. K. R. T., Kantha, L. H. \& CHOI, J. 2002 The loop current and adjacent rings delineated by Lagrangian analysis of the near-surface flow. J. Mar. Res. 60, 405-429.

LACKeY, T. C. \& SotiRopoulos, F. 2006 Relationship between stirring rate and Reynolds number in the chaotically advected steady flow in a container with exactly counter-rotating lids. Phys. Fluids 18 (5), 053601.

Ledwell, J. R., McGillicuddy, D. J. \& Anderson, L. A. 2008 Nutrient flux into an intense deep chlorophyll layer in a mode-water eddy. Deep-Sea Res. II 55, 1139-1160.

Lichtenberg, A. J. \& Lieberman, M. A. 1992 Regular and Chaotic Dynamics. Springer.

Lipphardt, B. L. Jr, Poje, A. C., Kirwan, A. D. Jr, Kantha, L. \& Zweng, M. 2008 Death of three loop current rings. J. Mar. Res. 66, 25-60.

Lipphardt, B. L. Jr, Small, D., Kirwan, A. D. Jr, Wiggins, S., Ide, K., Grosch, C. E. \& PADUAN, J. D. 2006 Synoptic Lagrangian maps: applications to surface transport in Monterey Bay. J. Mar. Res. 64, 221-247.

Lopez, J. M. 1998 Characteristics of endwall and sidewall boundary layers in a rotating cylinder with a differentially rotating endwall. J. Fluid Mech. 359, 49-79.

Lopez, J. M. \& MARQues, F. 2010 Sidewall boundary layer instabilities in a rapidly rotating cylinder driven by a differentially co-rotating lid. Phys. Fluids 22, 114109.

Lozier, S. M., Pratt, L. J., Rogerson, A. R. \& Miller, P. D. 1997 Exchange geometry revealed by float trajectories in the gulf stream. J. Phys. Oceanogr. 27, 2327-2341. 
MadAY, Y. \& PATERA, A. T. 1989 Spectral element methods for the Navier-Stokes equations. In State of the Art Surveys in Computational Mechanics (ed. A. K. Noor), pp. 71-143. ASME.

Malmotra, N. \& Wiggins, S. 1998 Geometric structues, lobe dynamics, and Lagrangian transport in flows with aperiodic time-dependence, with applications to Rossby wave flow. J. Nonlinear Sci. 8, 401-456.

Marshall, J. \& SchotT, F. 1999 Open-ocean convection: observations, theory and models. Rev. Geophys. 37, 1-64.

McGillicuddy, D. J., Anderson, L. A., Bates, N. R., Bibby, T., Buesseler, K. O., Carlson, C., Davis, C. S., Ewart, C., Falkowski, P. G., Goldthwait, S. A., Hansell, D. A., Jenkins, W. J., Johnson, R., Kosnyrev, V. K., Ledwell, J. R., Li, Q. P., Siegel, D. A. \& Steinberg, D. K. 2007 Eddy/wind interactions stimulate extraordinary mid-ocean plankton blooms. Science 316 (5827), 1021-1026 doi:10.1126/science. 1136256.

MeZIC, I. 2001 Chaotic advection in bounded Navier-Stokes flows. J. Fluid Mech. 431, 347-370.

Mezic, I. \& Wiggins, S. 1994 On the integrability of perturbations of three-dimensional fluid flows with symmetry. J. Nonlinear Sci. 4, 157-194.

Miller, P. D., Pratt, L. J., Helflrich, K. R. \& Jones, C. K. R. T. 2002 Chaotic transport of mass and potential vorticity for an island circulation. J. Phys. Oceanogr. 32, 80-102.

Mullowney, P., Julian, K. \& Meiss, J. D. 2005 Blinking rolls: chaotic advection in a 3D flow with an invariant. SIAM J. Appl. Dyn. Syst. 4, 159-186.

Mullowney, P., Julian, K. \& Meiss, J. D. 2008 Chaotic advection and the emergence of tori in the Kuppers-Lortz state. Chaos 18, 033104.

NGan, K. \& Shephard, T. G. 1999 A closer look at chaotic advection in the stratosphere. Part I: geometric structure. J. Atmos. Sci. 56, 4134-4152.

Ottino, J. M. 1989 The Kinematics of Mixing: Stretching, Chaos, and Transport. Cambridge University Press.

ÖZgÖKMEN, T. \& FischeR, P. 2008 On the role of bottom roughness in overflows. Ocean Model. 20, 336-361.

ÖZGÖKMEN, T. \& FISCHER, P. 2012 CFD application to oceanic mixed layer sampling with Lagrangian platforms. Intl J. Comput. Fluid. Dyn. 26, 337-348.

ÖZgöKmen, T. M., Fischer, P. F., DuAn, J. \& Iliescu, T. 2004a. Three-dimensional turbulent bottom density currents from a high-order nonhydrostatic spectral element model. J. Phys. Oceanogr. 34 (9), 2006-2026.

ÖzGökmen, T. M., Fischer, P. F., DuAn, J. \& Iliescu, T. 2004b. Entrainment in bottom gravity currents over complex topography from three-dimensional nonhydrostatic simulations. Geophys. Res. Lett. 31, L13212, doi:10.1029/2004GL020186.

ÖZgöKmen, T., Fischer, P. \& Johns, W. 2006 Product water mass formation by turbulent density currents from a high-order nonhydrostatic spectral element model. Ocean Model. 12, 237-267.

ÖZGÖKMEN, T., ILIESCU, T. \& FisCHER, P. $2009 a$ Large eddy simulation of stratified mixing in a three-dimensional lock-exchange system. Ocean Model. 26, 134-155.

ÖZGÖKMEN, T., ILIESCU, T. \& FISCHER, P. $2009 b$ Reynolds number dependence of mixing in a lock-exchange system from direct numerical and large eddy simulations. Ocean Model. 30, 190-206.

Özgökmen, T., Iliescu, T., Fischer, P., Srinivasan, A. \& Duan, J. 2007 Large eddy simulation of stratified mixing in two-dimensional dam-break problem in a rectangular enclosed domain. Ocean Model. 16, 106-140.

Özgökmen, T., Poje, A., Fischer, P., Childs, H., Krishnan, H., Garth, C., Haza, A. \& RYAN, E. 2012 On multi-scale dispersion under the influence of surface mixed layer instabilities and deep flows. Ocean Model. 56, 16-30.

Özgökmen, T., Poje, A., FIscher, P. \& HAZA, A. 2011 Large eddy simulations of mixed layer instabilities and sampling strategies. Ocean Model. 39, 311-331.

PATtANAyAK, A. K. 2001 Characterizing the metastable balance between chaos and diffusion. Physica D 148, 1-19. 
PAterA, A. 1984 A spectral element method for fluid dynamics; laminar flow in a channel expansion. J. Comput. Phys. 54, 468-488.

Pedlosky, T. \& Spall, M. A. 2005 Boundary intensification of vertical velocity in a $\beta$-plane basin. J. Phys. Oceanogr. 35, 2487-2500.

Poje, A. C. \& Haller, G. 1999 Geometry of cross-stream mixing in a double-gyre ocean model. J. Phys. Oceanogr. 29, 1649-1665.

Poje, A., Haza, A., Ozgokmen, T., Magaldi, M. \& Garraffo, Z. 2010 Resolution dependent relative dispersion statistics in a hierarchy of ocean models. Ocean Model. 31, 36-50.

Pratt, L. J., Lozier, M. S. \& Beliakova, N. 1995 Parcel trajectories in quasigeostrophic jets: neutal modes. J. Phys. Oceanogr. 25, 1451-1466.

Rogerson, A. M., Miller, P., Pratt, L. J. \& Jones, C. K. R. T. 1999 Lagrangian motion and fluid exchange in a barotropic meandering jet. J. Phys. Oceanogr. 29, 2635-2655.

Rypina, I. I., Brown, M. G., Beron-Vera, F. J., Kocak, H., Olascoaga, M. J. \& UdOVYDCHENKOV, I. A. $2007 a$ On the Lagrangian dynamics of atmospheric zonal jets and the permeability of the stratospheric polar vortex. J. Atmos. Sci. 64, 3593-3610.

Rypina, I. I., Brown, M. G., Beron-Vera, F. J., Kocak, H., Olascoaga, M. J. \& UdOVYDCHENKOV, I. A. $2007 b$ Robust transport barriers resulting from strong Kolmogorov-Arnold-Moser stability. Phys. Rev. Lett. 98, 104102. doi:10.1103/PhysRevLett.98. 10410.

RYPINA, I. I., BRown, M. G. \& KocAK, H. 2009 Transport in an idealized three-gyre system with an application to the adriatic sea. J. Phys. Oceanogr. 39, 675-690.

Rypina, I. I., PRATt, L. J. \& LozIER, S. M. 2011 Near-surface transport pathways in the North Atlantic Ocean. J. Phys. Oceanogr. 41, 911-925.

Rypina, I. I., Pratt, L. J., Pullen, J., Levin, J. \& Gordon, A. 2010 Chaotic advection in an archipelago. J. Phys. Oceanogr. 40, 1988-2006.

Rypina, I. I., SCOTt, S., Pratt, L. J. \& BRown, M. G. 2011 Investigating the connection between complexity of isolated trajectories and Lagrangian coherent structures. Nonlinear Proc. Geophys. 18, 977-987. doi:10.5194.

SAmelson, R. M. 1992 Fluid exchange across a meandering jet. J. Phys. Oceanogr. 22, 431-440.

SAmelson, R. M. \& Wiggins, S. 2006 Lagrangian Transport in Geophysical Jets and Waves: The Dynamical Systems Approach. Springer.

Shadden, S. C., Lekien, F., Paduan, J. D., Chavez, F. P. \& Marsden, J. E. 2009 Correlation between surface drifters and coherent structures from high-frequency radar data in Monterey Bay. Deep-Sea Res. II 56, 161-172.

Shott, F. M., Visbeck, M., Send, U., Fisher, J., Stramma, L. \& Desaubies, Y. 1996 Observations of deep convection in the Gulf of Lions, Northern Mediterranean, during the winter of 1991/92. J. Phys. Oceanogr. 26, 505-524.

Solomon, T. H. \& Mezic, I. 2003 Uniform resonant chaotic mixing in fluid flows. Nature 425, 376-380.

Sommeria, J. S., Meyers, D. \& Swinney, H. L. 1989 Laboratory models of a planetary eastward jet. Nature 337, 58-61.

Sundermeyer, M. A., Terray, E. A., Ledwell, J. R., Cunningham, A. G., LaRocque, P. E., BANIC, J. \& LillyCROP, W. J. 2007 Three-dimensional mapping of fluorescent dye using a scanning, depth-resolving airborne radar. J. Atmos. Ocean. Technol. 24, 1050-1065.

Thomas, L. N. \& Rhines, P. B. 2002 Nonlinear stratified spin up. J. Fluid Mech. 473, 211-244.

Yuan, G.-C., Pratt, L. J. \& Jones, C. K. R. T. 2004 Cross-jet transport and mixing in a 21/2-layer model. J. Phys. Oceangr. 34, 1991-2005.

Zaslavsky, G. M. \& Chirikov, B. V. 1972 Stochastic instability of non-linear oscillations. Sov. Phys. Uspekhi 14, 549-672. 